\title{
Isoparametric submanifolds and a Chevalley-type restriction theorem
}

\author{
Ernst Heintze, Xiaobo Liu, Carlos Olmos ${ }^{1}$
}

\begin{abstract}
We define and study isoparametric submanifolds of general ambient spaces and of arbitrary codimension. In particular we study their behaviour with respect to Riemannian submersions and their lift into a Hilbert space. These results are used to prove a Chevalley type restriction theorem which relates by restriction eigenfunctions of the Laplacian on a compact Riemannian manifold which contains an isoparametric submanifold with flat sections to eigenfunctions of the Laplacian of a section. A simple example of such an isoparametric foliation is given by the conjugacy classes of a compact Lie group and in that case the restriction theorem is a (well known) fundamental result in representation theory. As an application of the restriction theorem we show that isoparametric submanifolds with flat sections in compact symmetric spaces are level sets of eigenfunctions of the Laplacian and are hence related to representation theory. In addition we also get the following results. Isoparametric submanifolds in Hilbert space have globally flat normal bundle, and a general result about Riemannian submersions which says that focal distances do not change if a submanifold of the base is lifted to the total space.
\end{abstract}

\section{Introduction}

A hypersurface $M$ of a Riemannian manifold is called isoparametric, if its parallel manifolds have constant mean curvature or equivalently if $M$ is given (locally) as the regular level set of a function $f$ whose two parameters $\|g r a d f\|$ and $\Delta f$ are functions of $f$. The parallel manifolds of $M$ are also isoparametric and one gets (locally) two orthogonal foliations: The isoparametric foliation which consists of $M$ and its parallel

1 Research supported by Universidad Nacional de Córdoba, partially supported by CONICOR, Secyt-UNC and CIEM

1991 Mathematics Subject Classification. 53B25, 53C30, 53C40

Key words and phrases. Isoparametric submanifolds, Chevalley restriction theorem, polar actions, Riemannian submersions, representations of compact Lie groups, submanifolds of Hilbert space 
manifolds and the 1-dimensional foliation by the geodesics perpendicular to $M$. Typical examples are the principal orbits of cohomogeneity-1 actions. In spaces of constant curvature the definition implies that even the principal curvatures of $M$ are constant and not only their sum (E. Cartan). But of course this is no longer true in more general ambient manifolds.

The aim of this paper is twofold. Our first goal is to propose a definition for isoparametric submanifolds of arbitrary codimension which extends the above definition for hypersurfaces in a natural way. We call a submanifold $M$ to be isoparametric, if $\nu M$ is flat, the (close by) parallel manifolds have constant mean curvature in radial directions and if $M$ admits sections, that is, if for each $p \in M$ there exists a totally geodesic submanifold which is tangent to $\nu_{p} M$. Again one gets locally two transversal foliations, namely the isoparametric foliation by $M$ and its parallel manifolds and orthogonally the totally geodesic foliation by the sections. We study some properties of these submanifolds, in particular their behaviour with respect to Riemannian submersions. We also show that our definition coincides with that of Terng ([T1], [T2]), if the ambient manifold is a euclidean space, possibly an infinite dimensional Hilbert space. Furthermore we prove that a submanifold of a compact symmetric space is isoparametric with flat sections if and only if it is equifocal in the sense of Terng and Thorbergsson $[\mathrm{TT}]$.

Simple examples of isoparametric submanifolds are the (non-totally geodesic) leaves of a warped product. More interesting, the principal orbits of a polar action are isoparametric, where we call an isometric action to be polar, if the distribution of the normal spaces to the principal orbits is integrable. The integral manifolds of this distribution are necessarily totally geodesic and yield the sections. A typical example of a polar action is a compact Lie group $G$ with biinvariant metric which acts on itself by conjugation. The conjugacy classes of regular elements are the isoparametric submanifolds and the maximal tori are the sections in this case.

The linearized version of this example, namely the adjoint action of $G$ on its Lie algebra (which is also polar), brings us to our second topic, the Chevalley-type restriction theorem for isoparametric submanifolds. If $\mathfrak{g}$ denotes the Lie algebra of $G, \mathfrak{t} \subset \mathfrak{g}$ a maximal abelian subalgebra and $W$ the Weyl group, then one version of the classical Chevalley restriction theorem says (cf. [Hel2], Ch II, §5), that restricting polynomials from $\mathfrak{g}$ to $\mathfrak{t}$ defines an isomorphism from the space of $A d G$-invariant polynomials on $\mathfrak{g}$ onto the space of $W$-invariant polynomials on $\mathfrak{t}$ or equivalently from the space of polynomials on $\mathfrak{g}$ which are constant on the leaves of the isoparametric foliation onto the space of polynomials on $\mathfrak{t}$ which are constant on the intersections of the leaves with t. Now let $X$ be a compact symmetric or more generally normal homogeneous space and $M \subset X$ a compact isoparametric submanifold with flat sections whose parallel manifolds decompose $X$ (which is always the case if $X$ is simply connected). If $\Sigma$ is a section, then we have the following Chevalley-type restriction theorem (Theorem 7.1).

Theorem Restricting functions from $X$ to $\Sigma$ defines an isomorphism from $C^{\Delta}(X)^{M}$ 
onto $C^{\Delta}(\Sigma)^{M}$.

Here $C^{\Delta}(X)^{M}$ denotes the space of finite sums of eigenfunctions of the Laplacian of $X$ which are constant on $M$ and its parallel manifolds. Similarly $C^{\Delta}(\Sigma)^{M}$ denotes the space of finite sums of eigenfunctions of the Laplacian on $\Sigma$ which are constant on the intersections of $\Sigma$ with the parallel manifolds of $M$. The finite sums of eigenfunctions replace the polynomials in the classical restriction theorem. In fact, if one replaces $\mathfrak{g}$ and $\mathfrak{t}$ in this theorem by the corresponding unit spheres $S(\mathfrak{g})$ and $S(\mathfrak{t})$ - which are invariant under $G$ and $W$, respectively - then one gets the equivalent theorem that restriction defines an isomorphism from $C^{\Delta}(S(\mathfrak{g}))^{G}$ onto $C^{\Delta}(S(\mathfrak{t}))^{W}$. Recall that the finite sums of eigenfunctions of the Laplacian on the unit sphere are precisely the restrictions of the polynomials of the ambient euclidean space.

Even in the example above - the action of $G$ on itself by conjugation - the theorem is interesting, yet well known. In fact, it gives a weak form of the fundamental result of representation theory which says that restriction defines an isomorphism from $R(G)$ onto $R(T)^{W}$ where $T$ is a maximal torus and $R(G)$ and $R(T)$ denote the representation rings of $G$ and $T$, respectively. More precisely, it gives an isomorphism between the corresponding vector spaces $R(G) \otimes \mathbb{C}$ and $(R(T) \otimes \mathbb{C})^{W}$ (recall that the representation rings are $\mathbb{Z}$-modules).

If the ambient space $X$ in the theorem is in addition simply connected, then it follows moreover that $C^{\Delta}(X)^{M}$ is a polynomial algebra in $k=\operatorname{codim} M$ generators and that these generators can be chosen as eigenfunctions of the Laplacian of $X$ (Theorem 7.6). This in turn implies that isoparametric submanifolds with flat sections in compact, simply connected, normal homogeneous spaces are the simultanous level sets of eigenfunctions of the Laplacian (Corollary 7.7) and are hence related to representations of the isometry group of $X$. Recall, that the pull back of an eigenfunction of $X=G / H$ to $G$ is the coefficient of some representation of $G$. This result might be even interesting for isoparametric hypersurfaces of compact symmetric spaces and explains to some extent why all the known examples of isoparametric hypersurfaces in spheres are connected with representations, namely Clifford representations and isotropy representations of symmetric spaces.

One of our principal tools for the proof of the above results is the lifting of an isoparametric submanifold with flat sections in certain spaces to an isoparametric submanifold of a Hilbert space. This idea goes back to Terng and Thorbergsson [TT]. For the price of infinite dimensions, the ambient manifold is so to speak linearized and the theory of isoparametric submanifolds of Hilbert spaces (as developed by Terng in [T2]) becomes applicable. In particular one gets a formula for the mean curvature of the original isoparametric submanifold in this way ([KT]), which does not seem to be obtainable so far in a direct manner. This formula plays a key role for our results, as 
it relates the Laplacian of a section $\Sigma$ to the Laplacian of the ambient manifold $X$. We develop these ideas of Terng - Thorbergsson and King - Terng carefully in Chapter 5 and 6 and put them into a broader context. We also generalize a result of Terng Thorbergsson ([TT], Lemma 5.12) and O'Neill ([O'N2], Theorem 4) to the effect that for any Riemannian submersion $\pi: \hat{X} \rightarrow X$ focal distances and multiplicities of a submanifold $M$ of $X$ coincide with that of its lift $\hat{M}=\pi^{-1}(M) \subset \hat{X}$. This gives for example a simple geometric proof for the fact that fibres of a Riemannian submersion $\pi: V \rightarrow X$ are minimal (in a certain regularized sense), if $V$ is a Hilbert and $X$ a symmetric space.

To prove the equivalence of our notion of isoparametric submanifold with that of Terng for submanifolds of Hilbert spaces we essentially have to extend Cartan's theorem that isoparametric submanifolds have constant principal curvatures to infinite dimensions (Theorem 4.2). But there is also a more technical point, as Terng requires isoparametric submanifolds of Hilbert spaces to have globally flat normal bundle, that is with trivial, not only finite holonomy, and our definition is purely local. However, we show in Appendix B that global flatness is a consequence of her other conditions, like in finite dimensions.

Finally, we prove in Appendix A that our definition of polar actions (the distribution of normal spaces to the principal orbits is integrable) implies the existence of totally geodesic complete immersed sections through each point which meet all orbits and always perpendicularly. But in general these sections will neither be closed nor embedded (as required in the definition of [PT1]). Counterexamples are already given by certain cohomogeneity -1 actions on compact, simply connected symmetric spaces.

\section{Isoparametric submanifolds}

Let $M$ denote an immersed submanifold of a Riemannian manifold $N . M$ is said to have globally flat normal bundle, if the holonomy of the normal bundle is trivial, i.e. if any normal vector can be extended to a globally defined parallel normal field. It then follows in particular that $\nu M$ is flat. On the other hand, if $\nu M$ is flat, then there exists for each $p \in M$ an open neighborhood $U$ of $p$ in $M$, over which the normal bundle is globally flat. By eventually restricting $U$ further one can find an $r>0$ such that in addition the exponential map is a diffeomorphism on $\nu^{r} M_{\mid U}$, where $\nu^{r} M=\left\{\xi \in \nu_{M} \mid\|\xi\|<r\right\}$. The "local tube" $V:=\exp \left(\nu^{r} M_{\mid U}\right)$ around $M$ is then foliated by the parallel submanifolds $U_{\xi}=\{\exp \xi(q) \mid q \in U\}$, where $\xi$ is a parallel normal field along $U$ of length less than $r$. On $V \backslash U$ there exists the radial vector field $\frac{\partial}{\partial r}=\operatorname{grad} r$, where $r$ is the distance to $M$. It follows from the first variation formula that the restriction of $\frac{\partial}{\partial r}$ to any $U_{\xi}$ is a normal vector field. We say that locally the parallel submanifolds of $M$ have constant mean curvature in radial directions if for each 
$p \in M$ we can find $U$ and $r$ as above, such that the mean curvature in the direction of $\frac{\partial}{\partial r}$, i.e. $\operatorname{tr} A_{\frac{\partial}{\partial r}}$, is constant along $U_{\xi}$ for all parallel normal fields along $U$ with $\|\xi\| \in(0, r)$, where $A$ denotes the shape operator of $U_{\xi}$.

Definition $M$ is called almost isoparametric, if $\nu M$ is flat and if locally the parallel submanifolds of $M$ have constant mean curvature in radial directions.

Remark: It follows by continuity that an almost isoparametric submanifold has constant mean curvature in the direction of any parallel normal field, and thus has parallel mean curvature.

The condition on the mean curvature of parallel submanifolds can also be described in the following way.

Proposition 2.1 Let $M$ be an immersed submanifold with flat normal bundle. Then $M$ is almost isoparametric if and only if for each $p \in M$ there exists a local tube $V=\exp \left(\left.\nu^{r} M\right|_{U}\right)$ around $M$ such that for all parallel $\xi$ in $\nu^{r} M_{\mid U}$ the mapping $U \rightarrow$ $U_{\xi}, x \mapsto \exp \xi(x)$, is volume preserving up to a constant factor.

Proof: Let $\xi_{0}:=\xi\left(x_{0}\right)$ for some $x_{0} \in U$ and $Y_{1}, \ldots, Y_{m}(m=\operatorname{dim} M)$ a basis of $M$-Jacobi fields along $c_{\xi_{0}}$, i.e. of Jacobi fields associated to variations of $c_{\xi_{0}}$ through geodesics starting orthogonally from $M$, where $c_{v}$ is the geodesic in the direction of $v$. Then

$$
\left(\log \left\|Y_{1} \wedge \ldots \wedge Y_{m}\right\|\right)^{\prime}(t)=-\operatorname{tr} A_{\frac{\partial}{\partial r}\left(c_{\xi_{0}}(t)\right)},
$$

as one can choose the $Y_{i}$ in such a way that they are orthonormal at $t$ which implies

$$
\begin{aligned}
& \left(\log \left\|Y_{1} \wedge \ldots \wedge Y_{m}\right\|\right)^{\prime}(t)=\frac{1}{2}\left(\log \left\langle Y_{1} \wedge \ldots \wedge Y_{m}, Y_{1} \wedge \ldots \wedge Y_{m}\right\rangle\right)^{\prime}(t)= \\
& =\left\langle\left(Y_{1} \wedge \ldots \wedge Y_{m}\right)^{\prime}, Y_{1} \wedge \ldots \wedge Y_{m}\right\rangle(t)=\sum_{i}\left\langle Y_{i}^{\prime}, Y_{i}\right\rangle(t)= \\
& =-\sum_{i}\left\langle A_{\frac{\partial}{\partial r}} Y_{i}, Y_{i}\right\rangle .
\end{aligned}
$$

The last equality comes from the fact, that geodesics starting orthogonally from $U$ hit $U_{t \xi}$ orthogonally as well, so that the $Y_{i}$ are also $U_{t \xi^{-}}$Jacobi fields. Since the ratio of volume elements of $U_{t \xi_{0}}$ at $c_{\xi_{0}}(t)$ and $U$ at $x_{0}$ is given by the ratio of $\left\|Y_{1} \wedge \ldots \wedge Y_{m}\right\|$ at $t$ and 0 , respectively, the proposition follows from $(*)$.

In general the parallel submanifolds of an almost isoparametric submanifold need not be almost isoparametric themselfes. To remedy this situation we will require in addition that $M$ has totally geodesic sections. 
Definition An immersed submanifold $M$ is said to have (totally geodesic) sections if for each $p \in M$ there exists a totally geodesic submanifold $\Sigma$ (also called a section) which meets $M$ at $p$ orthogonally and whose dimension is equal to the codimension of $M$.

Obviously such a section has to coincide locally with $\exp \left(\nu_{p} M\right)$. Therefore $M$ has totally geodesic sections if and only if for each $p \in M, \exp \left(\nu_{p} M\right)$ is totally geodesic in a neighborhood of $p$. This condition is automatically satisfied if $M$ is a hypersurface or if $N$ has constant sectional curvature.

The following special case will be of interest later.

Definition $M$ is said to have flat sections if $M$ has totally geodesic sections which are flat with respect to the induced metric.

Besides hypersurfaces and submanifolds of euclidean spaces, interesting examples of submanifolds with flat sections are principal orbits of hyperpolar actions (cf. [HPTT] and the remark below).

Definition An immersed submanifold is called isoparametric if it is almost isoparametric and if it has totally geodesic sections.

The distinction between isoparametric and almost isoparametric is redundant if $M$ is a hypersurface or if the ambient manifold has constant curvature. Important examples of isoparametric submanifolds arise as the principal orbits of polar actions. We call (following a suggestion of W. Ziller) an isometric action of a Lie group on a Riemannian manifold to be polar if the distribution of the normal spaces to the principal orbits is integrable. The integral manifolds are then totally geodesic and yield the sections. If these are flat with respect to the induced metric, the action is called hyperpolar ([HPTT]). Recently A. Kollross [Ko] has classified hyperpolar actions on irreducible compact symmetric spaces and Podestà and Thorbergsson [PTh] have classified polar actions on compact rank 1 symmetric spaces. Our definition of polar is somewhat weaker than that of Palais and Terng [PT1] in that we do not require the existence of a closed embedded section. However, we prove in Appendix A that also with our definition sections (in complete manifolds) can be always extended to complete totally geodesic immersions which meet all orbits and always perpendicularly. But in general they are neither closed nor embedded. This shows for example the diagonal action of $S O(3)$ on $S_{r_{1}}^{2} \times S_{r_{2}}^{2}$ which is of cohomogeneity one and thus polar. But the geodesics normal to the principal orbits are not closed if the ratio $r_{1 / r_{2}}$ of the radii of the spheres is not rational.

The following two results show the strong influence of the existence of totally geodesic sections for submanifolds with flat normal bundle. 
Proposition 2.2 Let $M$ be a submanifold with globally flat normal bundle and assume that there exists $r>0$ such that $\exp _{\mid \nu^{r} M}$ is a diffeomorphism and $\exp \left(\nu_{p}^{r} M\right)$ is totally geodesic for all $p \in M$. Then the parallel manifolds $M_{\xi},\|\xi\|<r$, meet the sections $\exp \left(\nu_{p}^{r} M\right)$ orthogonally as well and have globally flat normal bundle, too. In fact, a parallel normal field along $M$ transported to $M_{\xi}$ by parallel translation along the geodesics $\exp t \xi$ is a parallel normal field along $M_{\xi}$.

Remark: It follows in particular that the radial vector field $\frac{\partial}{\partial r}$ is parallel in the normal bundle of any $M_{\xi}, 0<\|\xi\|<r$.

\section{Proof:}

(i) Let $\xi$ be a parallel normal field along $M$ with $\|\xi\|<r$ and fix some $p \in M$. Let $c(t):=\exp t \xi(p)$ and $q:=c(1) \in M_{\xi}$. If $v \in T_{p} M$ and $\gamma$ is a curve in $M$ with $\dot{\gamma}(0)=v$, then $\alpha(s, t):=\exp t \xi(\gamma(s))$ is a variation of $c$ through geodesics with $\alpha(s, 0)=\gamma(s)$ and $\alpha(s, 1) \in M_{\xi}$. The variation vector field $Y_{v}(t):=\frac{\partial \alpha}{\partial s}(0, t)$ is the Jacobi field along $c$ with $Y_{v}(0)=v$ and $Y_{v}^{\prime}(0)=\frac{D}{\partial t} \frac{\partial \alpha}{\partial s}(0,0)=\frac{D}{\partial s} \frac{\partial \alpha}{\partial t}(0,0)=\xi^{\prime}(0)=-A_{\xi} v \in T_{p} M$. Since $T_{q} M_{\xi}=\left\{Y_{v}(1) \mid v \in T_{p} M\right\}$, we have to show that these Jacobi fields $Y_{v}$ are perpendicular to the section $\Sigma:=\exp \nu_{p} M$. But this follows from the fact that $Y_{v}$ is a solution of $Y^{\prime \prime}+R_{\dot{c}} Y=0$ with initial conditions in $\left(T_{\dot{c}(0)} \Sigma\right)^{\perp}$, where $R_{\dot{c}}=R(., \dot{c}) \dot{c}$, and that $T_{\dot{c}(0)} \Sigma$ as well as $\left(T_{\dot{c}(t)} \Sigma\right)^{\perp}$ are families of parallel subspaces along $c(t)$ which are invariant under $R_{\dot{c}}$. Therefore $M_{\xi}$ meets $\Sigma$ perpendicularly.

(ii) Let $\eta$ be another parallel normal field along $M$ and $\eta^{*}$ be the vector field along $M_{\xi}$ which is obtained by parallel translating $\eta(p)$ along $\exp t \xi(p)$ to $\exp \xi(p)$. Then $\eta^{*}$ is a normal field along $M$ by the above considerations. Let $p \in M$ and $\gamma(s)$ a curve in $M$ starting at $p$ and $\alpha(s, t)=\exp t \xi(\gamma(s))$. Let $\Sigma=\exp _{p} \nu_{p} M$ as above and $\eta(s, t)$ be the parallel translation of $\eta(\gamma(s))$ along $\tau \rightarrow \exp \tau \xi(\gamma(s))$ up to $\exp t \xi(\gamma(s))$, so that $\eta(s, 0)=\eta(\gamma(s))$ and $\eta(s, 1)=\eta^{*}(\exp \xi \gamma(s))$. If $v(t)$ is parallel along $c(t)$ and tangent to $\Sigma$, then $\frac{\partial}{\partial t}\left\langle\frac{D \eta}{\partial s}, v\right\rangle_{/ s=0}=\left\langle\frac{D}{\partial t} \frac{D \eta}{\partial s}, v\right\rangle_{/ s=0}=\left\langle R\left(\dot{c}, \frac{\partial \alpha}{\partial s}\right) \eta, v\right\rangle_{/ s=0}=0$, since $\frac{\partial \alpha}{\partial s} /_{s=0} \perp \Sigma$ and $\dot{c}, \eta, v$ are tangent to $\Sigma$. Since $\left\langle\frac{D \eta}{\partial s}, v\right\rangle_{/ s=0}=0$ at $t=0, \frac{D \eta}{\partial s} /_{s=0}$ is perpendicular to $\Sigma$ for all $t \in[0,1]$, in particular for $t=1$, which shows that $\eta^{*}$ parallel in $\nu M_{\xi}$.

Proposition 2.3 The following conditions on an immersed submanifold $M$ of $N$ are equivalent.

(i) $M$ has flat normal bundle and totally geodesic sections.

(ii) Locally, (i.e. for each $p \in M$ there exists a neighborhood in $N$, such that in this neighborhood) $M$ is the fibre of a Riemannian submersion $f: N \rightarrow B$ with integrable horizontal spaces.

(iii) Locally, $M$ is the leaf of a foliation which admits an orthogonal, transversal and totally geodesic foliation.

(iv) Locally, $N$ splits as $N=N_{1} \times M$ with metric $g_{1} \oplus g_{2}(x)$, where $g_{1}$ is a fixed metric on $N_{1}$ and $g_{2}(x)$ is a metric on $M$ which depends on $x \in N_{1}$. 
Proof: "(i) $\Rightarrow$ (iii)" follows from Proposition 2.2., "(iv) $\Rightarrow$ (ii)" is obvious, as the projection onto $N_{1}$ is a Riemannian submersion. "(ii) $\Rightarrow$ (i)" follows from the remark that the O'Neill tensor of $f: N \rightarrow B$ vanishes, and Lemma 3.1 below. Thus we are left to show "(iii) $\Rightarrow$ (iv)". Since the foliations are transversal, $N$ is locally diffeomorphic to $N_{1} \times M$ and the $N_{1}$ factors are by assumption totally geodesic and perpendicular to the $M$-factors. If $c_{1}$ and $c_{2}$ are differentiable curves in $N_{1}$ and $M$, respectively and $\alpha(s, t):=\left(c_{1}(s), c_{2}(t)\right)$, we therefore have $\frac{1}{2} \frac{\partial}{\partial t}\left\|\frac{\partial \alpha}{\partial s}\right\|^{2}=\left\langle\frac{D}{\partial t} \frac{\partial \alpha}{\partial s}, \frac{\partial \alpha}{\partial s}\right\rangle=\left\langle\frac{D}{\partial s} \frac{\partial \alpha}{\partial t}, \frac{\partial \alpha}{\partial s}\right\rangle=$ $\frac{\partial}{\partial s}\left\langle\frac{\partial \alpha}{\partial t}, \frac{\partial \alpha}{\partial s}\right\rangle-\left\langle\frac{\partial \alpha}{\partial t}, \frac{D}{\partial s} \frac{\partial \alpha}{\partial s}\right\rangle=0$. Hence the metric on the $N_{1}$-factors is constant.

The following theorem shows that for an isoparametric submanifold the mean curvature of the parallel submanifolds is not only constant in radial directions but also in the direction of any parallel normal field. More precisely we have:

Theorem 2.4 Let $M$ be an immersed submanifold of $N$ with flat normal bundle and totally geodesic sections. Then $M$ is isoparametric if and only if locally the parallel submanifolds have parallel mean curvature.

Proof: We may assume that $M$ has globally flat normal bundle and there exists $r>0$ such that exp is a diffeomorphism on $\nu^{r} M$ and $\exp \nu_{p}^{r} M$ is totally geodesic for all $p \in M$. If the close by parallel manifolds $M$ have parallel mean curvature, then they have constant mean curvature in the radial direction $\frac{\partial}{\partial r}$, as $\frac{\partial}{\partial r}$ is parallel in $\nu M_{\xi}$ by the remark following Proposition 2.2. Hence $M$ is isoparametric.

On the other hand, let $M$ be isoparametric and let $\eta$ be the vector field on $\exp \nu^{r} M$ whose restriction to any parallel manifold $M_{\xi}$ is the mean curvature vector field of $M_{\xi}$. By Proposition 2.4 we may assume that $\exp \nu^{r} M=N_{1} \times M$ with metric $g_{1} \oplus g_{2}(x), x \in$ $N_{1}$, and that $N_{1}=B_{r}(0) \subset \nu_{p} M$. We then have to show that $\eta=\eta(x, y)$, where $x \in N_{1}$ and $y \in M$, is constant in $y$. By eventually restricting $M$ further we may assume that there exist on $M$ globally defined vector fields $X_{1}, \ldots, X_{m}$ which form at every point a basis of the tangent space. We view these also as vector fields on $N_{1} \times M$ which are tangent to $M$ and constant in the $N_{1}$-direction. For any $\xi \in T_{(x, y)} N_{1} \times M$ which is normal to $M$ we then have as in the proof of Proposition 2.1

$$
\operatorname{tr} A_{\xi}=-\xi \log \left\|X_{1} \wedge \ldots \wedge X_{m}\right\|
$$

where $A_{\xi}$ is the shape operator of $M$. Hence $\eta=\operatorname{grad}_{N_{1}} f$, where $f=-\log \| X_{1} \wedge$ $\ldots \wedge X_{m} \|$ and where the subscript $N_{1}$ denotes the orthogonal projection onto $T N_{1}$. If $X$ and $Y$ are vector fields on $N_{1} \times M$ with $X$ tangent to $M$ and constant in the $N_{1}$ direction and $Y$ tangent to $N_{1}$ and constant in the $M$-direction, then $[X, Y]=0$ and $X\langle\eta, Y\rangle=X(Y f)=Y(X f)$. If $Y$ is the radial vector field, then $\langle\eta, Y\rangle=\operatorname{tr} A_{y}$ is constant in the direction of $X$ by assumption. Hence $X f$ is constant in radial directions and thus constant on any section $N_{1} \times\{q\}, q \in M$. This implies $Y(X f)=0$ for any $Y$ as above and hence $X\langle\eta, Y\rangle=0$. Thus $\eta=\eta(x, y)$ is constant in $y$. 
From Proposition 2.3 and the theorem we get:

Corollary 2.5 The locally defined parallel manifolds of an isoparametric submanifold are isoparametric as well, and thus define locally an isoparametric foliation.

Together with Proposition 2.1 we get:

Corollary 2.6 An immersed submanifold $M$ is isoparametric if and only if $\nu M$ is flat, $M$ has totally geodesic sections and if the projection from $M$ to any (sufficiently close) parallel submanifold along the sections is volume preserving up to a constant factor.

In particular the leaves of a warped product $N \times_{f} M, f: N \rightarrow \mathbb{R}$, more precisely the factors $\{n\} \times M$ are isoparametric, as the projection from one such leaf to another is even an isometry up to a constant factor and the factors $N \times\{m\}$ are totally geodesic.

We finally discuss the relation of our definition to some of the previous ones. The classical definition used by Segre, Levi-Civita and E. Cartan (cf. [Ca]) calls a hypersurface $M$ of a space form $N$ to be isoparametric if it is given as a regular level set of a function $f: N \rightarrow \mathbb{R}$ whose two "parameters" $\|\operatorname{grad} f\|$ and $\Delta f$ are functions of $f$. Geometrically this means precisely that parallel submanifolds have constant mean curvature (and that the normal bundle is globally flat). Thus the definition is essentially equivalent to ours. Cartan had shown that a hypersurface of a space form is isoparametric if and only if it has constant principal curvatures. Terng [T1] used this result to define a submanifold of arbitrary codimension of a space form to be isoparametric if it has flat normal bundle and if the principal curvatures in the direction of any (locally defined) parallel normal field are constant. It follows from her extension of Cartan's result above, that for submanifolds of space forms this definition is equivalent to ours. She also extended her definition to submanifolds in Hilbert space in [T2] and we will prove (Corollary 4.3) that also in this case both definitions coincide, by extending Cartan's result further. On the other hand, Wang had already shown in [W] that an isoparametric hypersurface in $\mathbb{C} P^{n}$ does not need to have constant principal curvatures. He constructs an example by pushing down a certain non-homogeneous isoparametric hypersurface in $S^{2 n+1}$ via the Hopf fibration into $\mathbb{C} P^{n}$. This shows also that the property of having constant principal curvatures for a hypersurface is not preserved under Riemannian submersions. Another definition of isoparametric submanifolds, but with the restriction $\operatorname{dim} M \geq \frac{1}{2} \operatorname{dim} N$ and $\nu M$ globally flat, had been given by Carter and West $[\mathrm{CW}$ ] using differential forms and the Hodge staroperator. Their Theorems (1.1) to (2.5) show that it is equivalent to ours under the restrictions made. Finally we mention that already Harle had given a definition of isoparametric submanifold in [Ha] which is very similar to ours, but had applied it only to submanifolds of space forms. We would like to thank G. Thorbergsson for pointing out this reference to us. 


\section{Isoparametric submanifolds and Riemannian submersions}

In later sections we will make strong use of the fact, that isoparametric submanifolds behave nicely with respect to Riemannian submersions. Let $\pi: X \rightarrow B$ be a Riemannian submersion. The tangent bundle of $X$ splits orthogonally into the vertical bundle $\mathcal{V}$ (which is tangent to the fibres) and the horizontal bundle $\mathcal{H}=\mathcal{V}^{\perp}$. Let $\nabla$ and $\bar{\nabla}$ be the covariant derivatives of $X$ and $B$, respectively. Then we have for all vector fields $V, W$ on $B$

$$
\nabla_{\hat{V}} \hat{W}=\bar{\nabla}_{V} W+O^{\prime} N(\hat{V}, \hat{W})
$$

where ${ }^{\wedge}$ denotes the horizontal lift and $O^{\prime} N(\hat{V}, \hat{W}):=\left(\nabla_{\hat{V}} \hat{W}\right)^{\mathcal{V}}$ is one of the tensors of O'Neill ([O'N]), usually denoted by A. Since also $O^{\prime} N(\hat{V}, \hat{W})=\frac{1}{2}[\hat{V}, \hat{W}]^{\mathcal{V}}$, this tensor vanishes precisely, if the horizontal distribution is integrabel and hence totally geodesic. The curvature $\bar{K}(\sigma)$ of a plane $\sigma$ tangent to $B$ is related to the curvature $K(\hat{\sigma})$ of a horizontal lift by

$$
\bar{K}(\sigma)=K(\hat{\sigma})+3\left\|O^{\prime} N(\hat{v}, \hat{w})\right\|^{2},
$$

where $v, w$ is an orthonormal base of $\sigma$. Thus these two curvatures coincide if and only if $O^{\prime} N$ vanishes on $\hat{\sigma}$.

The vanishing of $O^{\prime} N$ can also be described as follows.

Lemma 3.1 Let $b \in B$. Then $O^{\prime} N$ vanishes along the fibre $F_{b}:=\pi^{-1}(b)$ if and only if for each $v \in T_{b} B$ the horizontal lift $\hat{v}$ of $v$ is parallel in $\nu F_{b}$.

Proof: We extend $v$ locally to a vector field which we also denote by $v$. Then $\hat{v}$ is parallel in $\nu F_{b}$ if and only if $\left\langle\nabla_{Y} \hat{v}, \hat{w}\right\rangle=0$ for all vertical vector fields $Y$ and all horizontal lifts $\hat{w}$ of vectors $w \in T_{b} B$. Since $Y$ is $\pi$-related to zero and $\hat{v}$ to $v,[Y, \hat{v}]$ is $\pi$-related to zero, i.e. vertical. Thus $\left\langle\nabla_{Y} \hat{v}, \hat{w}\right\rangle=\left\langle\nabla_{\hat{v}} Y, \hat{w}\right\rangle=-\left\langle Y, \nabla_{\hat{v}} \hat{w}\right\rangle=-\left\langle Y, O^{\prime} N(\hat{v}, \hat{w})\right\rangle$, from which the lemma follows.

The following Proposition generalizes a result of Wang ([W], Corollary 2) from hypersurfaces to submanifolds of arbitrary codimension.

Proposition 3.2 Let $\pi: X \rightarrow B$ be a Riemannian submersion with minimal fibres and $M \subset B$ an embedded submanifold. Let $M^{*}:=\pi^{-1}(M)$ and assume that $O^{\prime} N=0$ on $\nu M^{*}$. Then $M$ is almost isoparametric if and only if $M^{*}$ is almost isoparametric.

\section{Remarks:}

(i) Since $M^{*}$ contains with any point the entire fibre through the point, its normal spaces are horizontal and are mapped isometrically by $\pi_{*}$ onto the normal spaces of $M$. In particular $M^{*}$ has the same codimension as $M$. 
(ii) If $M$ is a hypersurface, so is $M^{*}$ and the condition on $O^{\prime} N$ is automatically satisfied as $\mathrm{O}^{\prime} \mathrm{N}(X, X)=0$ for all $X$.

Proof: It follows from $O^{\prime} N=0$ on $\nu M^{*}$ and the proof of Lemma 3.1 that the horizontal lift $\hat{\xi}$ of a normal vector field $\xi$ is parallel in $\nu M^{*}$ in vertical directions. Since $\left(\nabla_{\hat{v}} \hat{\xi}\right)^{\perp}=$ $\left(\widehat{\nabla_{v} \xi}\right)^{\perp}$ for any $v \in T B, \hat{\xi}$ is also parallel in $\nu M^{*}$ in horizontal directions if and only if $\xi$ is parallel in $\nu M$. Hence $\nu M^{*}$ is flat if and only if $\nu M$ is flat and in that case the horizontal lifts of parallel normal fields are precisely the parallel normally fields of $M^{*}$ locally.

Let $\nu M$ be flat and $V=\exp \left(\nu^{r} M_{\mid U}\right)$ a local tube around $M$. Let $\xi \in \nu^{r} M_{\mid U}$ be parallel, $U^{*}=\pi^{-1}(U)$ and $U_{\xi}^{*}:=\pi^{-1}\left(U_{\xi}\right)$. Since $\pi(\exp t \hat{\xi})=\exp t \xi$, the parallel manifold $\left(U^{*}\right)_{\hat{\xi}}$ of $U^{*}$ is contained in $U_{\xi}^{*}$. But actually equality $\left(U^{*}\right)_{\hat{\xi}}=U_{\xi}^{*}$ holds, as one can reverse the roles of $U$ and $U_{\xi}$ for this argument: $U^{*}$ is obtained from $\left(U^{*}\right)_{\hat{\xi}}$ by lifting the geodesics $\exp t \xi$ from $U$ to $U_{\xi}$ with the reversed direction horizontally. The proposition follows therefore from the following lemma.

Lemma 3.3 Let $\pi: X \rightarrow B$ be a Riemannian submersion with minimal fibres, $M \subset B$ be an embedded submanifold and $M^{*}:=\pi^{-1}(M)$. Then the mean curvature vector field of $M^{*}$ is the horizontal lift of that of $M$. In particular $\operatorname{tr} A_{\hat{\xi}}^{*}=\operatorname{tr} A_{\xi}$ for any normal vector $\xi$ of $M$, where $\hat{\xi}$ is a horizontal lift and $A, A^{*}$ denote the shape operators of $M$ and $M^{*}$, respectively.

Proof: Let $\hat{b} \in X$ and $b:=\pi(\hat{b})$. We choose an orthonormal basis $e_{1}, \ldots, e_{m} \in T_{b} M$ and extend their horizontal lifts $\hat{e}_{1}, \ldots, \hat{e}_{m}$ by an orthonormal basis $f_{1}, \ldots, f_{k}$ of $T_{\hat{b}}\left(\pi^{-1}(b)\right)$ to one of $T_{\hat{b}} M^{*}$. Then $\eta^{*}=\sum_{i} \alpha^{*}\left(\hat{e}_{i}, \hat{e}_{i}\right)+\sum_{j} \alpha^{*}\left(f_{j}, f_{j}\right)=\sum_{i} \alpha^{*}\left(e_{i}, e_{i}\right)$, where $\eta^{*}$ denotes the mean curvature vector field and $\alpha^{*}$ the second fundamental form of $M^{*}$. Since $\left\langle\alpha^{*}\left(\hat{e}_{i}, \hat{e}_{i}\right), \hat{\xi}\right\rangle=\left\langle\nabla_{\hat{e}_{i}} \hat{e}_{i}, \hat{\xi}\right\rangle=\left\langle\bar{\nabla}_{e_{i}} e_{i}, \xi\right\rangle=\left\langle\alpha\left(e_{i}, e_{i}\right), \xi\right\rangle$ for any $\xi \in \nu_{b} M$ the lemma follows.

Remark: If $\nu M$ is globally flat in Proposition 3.2, so is $\nu M^{*}$, since the horizontal lift of a parallel normal field is a parallel normal field of $M^{*}$. The converse is not always true, but is true, if the fibres are connected. In that case the restriction of a parallel normal field $\xi^{*}$ in $\nu M^{*}$ to a fibre is not only locally a horizontal lift of a normal vector of $M$, but also globally, and $\xi^{*}$ can be pushed down to a globally defined parallel normal field of $M$.

Theorem 3.4 Let $\pi: X \rightarrow B$ be a Riemannian submersion with minimal fibres, $M \subset B$ be a submanifold and $M^{*}:=\pi^{-1}(M)$. Then the following conditions are equivalent.

(i) $M^{*}$ is isoparametric with horizontal sections, i.e. sections which are perpendicular to the fibres. 
(ii) $M$ is isoparametric and $O^{\prime} N=0$ on all horizontal lifts of tangent vectors to sections of $M$.

Proof: A horizontal section $\Sigma^{*}$ of $M^{*}$ is mapped under $\pi$ isometrically onto a section of $M$, as geodesics in $\Sigma^{*}$ are horizontal and are thus mapped to geodesics in $M$. From O'Neill's curvature formula it thus follows that $O^{\prime} N=0$ on tangent vectors to $\Sigma^{*}$. Together with Proposition 3.2 this proves "(i) $\Rightarrow$ (ii)".

If (ii) holds then $M^{*}$ is almost isoparametric and its parallel manifolds are horizontal lifts of parallel manifolds of $M$ (locally) by Proposition 3.2 and its proof. The normal distribution $\mathcal{D}$ to the parallel manifolds of $M^{*}$ is therefore horizontal. It is also integrabel and totally geodesic, since we have for any $\xi, \eta \in \mathcal{D}_{p} \quad\left(\nabla_{\xi} \eta\right)^{\mathcal{V}}=0$ by the assumption on $O^{\prime} N$ and $\left\langle\nabla_{\xi} \eta, \hat{v}\right\rangle=\left\langle\bar{\nabla}_{\pi_{*} \xi} \pi_{*} \eta, v\right\rangle=0$ for any $v$ which is tangent to the parallel submanifold of $M$ through $\pi(p)$. Thus (i) follows.

Corollary 3.5 Let $\pi: X \rightarrow B$ be a Riemannian submersion with minimal fibres and let $X$ have non negative curvature. If $M \subset B$ is an isoparametric submanifold with flat sections, so is $M^{*}=\pi^{-1}(M)$.

Proof: By O'Neill's curvature formula, condition (ii) of the theorem is satisfied.

Remark: The Corollary as well as Proposition 3.2 and Theorem 3.4 are also true if $X$ is a separable Hilbert space. In fact, we will show in section 5 that the mean curvature of $M^{*}$ can be defined also in this situation and in such a way that Lemma 3.3 is valid. But then the same arguments apply.

In a special situation also the converse to Corollary 3.5 holds.

Corollary 3.6 Let $G$ be a Lie group with biinvariant metric, $H \subset G$ a closed subgroup and $G / H$ the normal homogeneous space endowed with the quotient metric, so that $\pi: G \rightarrow G / H$ is a Riemannian submersion. Then a submanifold $M$ of $G / H$ is isoparametric with flat sections if and only if $M^{*}=\pi^{-1}(M)$ is isoparametric with flat sections.

Proof: Let $M^{*}$ be isoparametric with flat sections and assume for convenience that $e \in M^{*}$. Then a section at $e$ is an abelian Lie group $A$ which is perpendicular to the fibre $H$. Since the metric is invariant under left translations, $A$ is also perpendicular to the fibres $a \cdot H, a \in A$. Hence the first condition of the theorem is satisfied and $M$ is isoparametric with flat sections.

The other direction follows from Corollary 3.5. 


\section{Isoparametric submanifolds of Hilbert space}

In this section we extend our definition of isoparametric submanifolds to the situation where the ambient manifold is a Hilbert space and show that it coincides with that of Terng in [T2]. This may be viewed as a generalization of theorems of Cartan and Terng to the infinite dimensional case.

We will always (in the whole paper) denote by $V$ a real separable Hilbert space. We begin by defining the regularized trace of certain compact operators, which will later be the shape operators of submanifolds of $V$. Our regularization is somewhat different from the $\zeta$-regularization used by King and Terng $[\mathrm{KT}]$ and has the advantage of being easier to handle. In all relevant cases, however, both regularizations will coincide (cf. the remark below).

Definition Let $A: V \rightarrow V$ be a compact selfadjoint operator with non-zero eigenvalues $\mu_{1} \leq \mu_{2} \leq \ldots<0<\ldots \leq \lambda_{2} \leq \lambda_{1}$, repeated with multiplicities. Then $A$ is called regularizable if $\operatorname{tr} A^{2}<\infty$ and $\sum_{1}^{\infty}\left(\lambda_{k}+\mu_{k}\right)$ converges where $\lambda_{k}$ or $\mu_{k}$ is understood to be zero, if there are less than $k$ positive or negative eigenvalues, respectively. If $A$ is regularizable, then

$$
\operatorname{tr}_{r} A:=\sum_{1}^{\infty}\left(\lambda_{k}+\mu_{k}\right)
$$

is called the regularized trace of $A$.

In general $\Sigma \lambda_{k}$ and $\Sigma \mu_{k}$ will not converge. A typical example is $\lambda_{k}=-\mu_{k}=1 / k$ with $\operatorname{tr}_{r} A=0$. The definition of the regularized trace therefore depends strongly on the chosen order of the eigenvalues.

Remark: King and Terng call a compact, self-adjoint operator $A: V \rightarrow V$ with nonzero eigenvalues $\mu_{1} \leq \mu_{2} \leq \ldots<0<\ldots \leq \lambda_{2} \leq \lambda_{1} \quad \zeta$-regularizable if $\sum_{k} \lambda_{k}^{s}-\sum_{k}\left|\mu_{k}\right|^{s}$ converges for all $s>1$ and $\operatorname{tr}_{\zeta} A:=\lim _{s \searrow 1}\left(\sum_{k} \lambda_{k}^{s}-\sum_{k}\left|\mu_{k}\right|^{s}\right)$ exists. By the dominated convergence theorem, it follows easily that $\operatorname{tr}_{\zeta} A=\operatorname{tr}_{r} A$, if $A$ is $\zeta$-regularizable and $\sum_{k}\left(\lambda_{k}+\mu_{k}\right)$ converges absolutely. In fact, $\lim _{s \searrow 1} \sum_{k}\left(\lambda_{k}^{s}-\left|\mu_{k}\right|^{s}\right)=\sum_{k} \lim _{s \searrow 1}\left(\lambda_{k}^{s}-\left|\mu_{k}\right|^{s}\right)=$ $\sum_{k}\left(\lambda_{k}+\mu_{k}\right)$, as $\left|x^{s}-y^{s}\right| \leq 2|x-y|$ for all $s \in[1,2]$ and $x, y \in[0,1]$.

Definition An immersed submanifold $M$ of $V$ is called regularizable, if for each $\xi \in \nu M$ the shape operator $A_{\xi}$ is regularizable. In this case $t_{r} A_{\xi}$ is also called the mean curvature of $M$ in direction of $\xi$. 
The following lemma implies that parallel manifolds of $M$ are regularizable in radial directions, if $M$ is regularizable. It also motivates the condition $\operatorname{tr} A^{2}<\infty$ in the definition of regularizability.

Lemma 4.1 Let $\left\{a_{k}\right\}_{k \in \mathbb{N}}$ be a sequence of complex numbers such that $\Sigma a_{k}$ and $\Sigma\left|a_{k}\right|^{2}$ converge. Then also $\Sigma \frac{a_{k}}{1-a_{k} z}$ converges for all $z \in \mathbb{C}$ for which $a_{k} z \neq 1$ for all $k \in \mathbb{N}$. The limit function $f$ is meromorphic in $\mathbb{C}$ and $\left\{1 / a_{k} \mid k \in \mathbb{N}, a_{k} \neq 0\right\}$ is the set of its poles. The residue of $f$ at a pole $1 / a_{k}$ is equal to $-\sharp\left\{\ell \in \mathbb{N} \mid a_{\ell}=a_{k}\right\}$. In particular, $f$ determines the non-zero $a_{k}^{\prime} s$ together with their multiplicities.

Proof: Given $R>0$ there exists $k_{0} \in \mathbb{N}$ with $\left|a_{k}\right| \leq \frac{1}{2 R}$ for all $k \geq k_{0}$ and hence $\left|1-a_{k} z\right| \geq \frac{1}{2}$ in the closed ball of radius $R$ around zero. Therefore $\sum_{k_{0}}^{\infty} \frac{a_{k}}{1-a_{k} z}=$ $\sum_{k_{0}}^{\infty} a_{k}+\sum_{k_{0}}^{\infty} \frac{a_{k}^{2} z}{1-a_{k} z}$ converges in this ball uniformely to a holomorphic function. The other statements are now obvious.

If $M$ is a regularizable submanifold of a Hilbert space $V$ and $\xi$ is a parallel normal field along $M$, then, as in finite dimensions, $M_{t \xi}:=\{p+t \xi(p) \mid p \in M\}$ is, at least locally and for small $t$, a submanifold of $V$ diffeomorphic to $M$. In fact, $p \mapsto p+t \xi(p)$ has differential $i d-t A_{\xi(p)}$ and is thus for any $p \in M$ injective in some neighborhood of $p$, if $t$ is small. Moreover the tangent spaces of $M$ and $M_{t \xi}$ coincide in corresponding points so that $\xi_{t}^{*}$, which is defined to be the parallel translation of $\xi(p)$ to $p+t \xi(p)$, is a parallel normal field along $M_{t \xi}$. Again as in finite dimensions the eigenvalues of the shape operator of $M_{t \xi}$ in direction of $\xi_{t}^{*}$ are $\frac{\lambda_{k}}{1-t \lambda_{k}}$ and $\frac{\mu_{k}}{1-t \mu_{k}}$, respectively, where $\mu_{1} \leq \mu_{2} \leq \ldots \leq 0 \leq \lambda_{2} \leq \lambda_{1}$ are the eigenvalues of $A_{\xi}$ and $t$ is sufficiently small. But then $\frac{\mu_{1}}{1-t \mu_{1}} \leq \frac{\mu_{2}}{1-t \mu_{2}} \leq \ldots \leq 0 \leq \ldots \leq \frac{\lambda_{2}}{1-t \lambda_{2}} \leq \frac{\lambda_{1}}{1-t \lambda_{1}}$ due to the monotonicity of the function $x \mapsto \frac{x}{1-t x}$. Hence the regularized mean curvature of $M_{t \xi}$ in direction of $\xi_{t}^{*}$ is defined by Lemma 4.1. Moreover, if this is constant along $M_{t \xi}$ for small $t$, then Lemma 4.1 shows that the non-zero eigenvalues of $A_{\xi}$ together with their multiplicities are constant along $M$.

Definition Let $M$ be an immersed submanifold of a separable Hilbert space. $M$ is called isoparametric, if $M$ is regularizable, $\nu M$ is flat and if for each $p \in M$ there exists a neighborhood in which the parallel manifolds $M_{\xi}$ have constant mean curvature in direction $\xi^{*}$ (which is the parallel translation of $\xi$ to $M_{\xi}$ and thus a constant multiple of the radial direction).

The following result generalizes the characterization of Cartan and Terng of isoparametric submanifolds in euclidean space to infinite dimensions.

Theorem 4.2 Let $M$ be an immersed regularizable submanifold of a separable Hilbert space with flat normal bundle. Then the following conditions are equivalent: 
(i) $M$ is isoparametric.

(ii) If $\xi$ is a parallel normal field in an open connected subset $U$ of $M$ then the shape operators $A_{\xi(p)}$ and $A_{\xi(q)}$ are orthogonally equivalent for all $p, q, \in U$.

Proof: Let (iii) be the statement: If $\xi$ is a parallel normal field in an open connected subset $U$ of $M$ then the non-zero eigenvalues of $A_{\xi}$ together with their multiplicities are constant on $U$.

Lemma 4.1 together with the discussion above proves (i) $\Rightarrow$ (iii). Vice versa, (i) is a trivial consequence of (iii) since the mean curvature of $M_{\xi}$ in the direction of $\xi^{*}$ is given by $\sum_{k}\left(\frac{\lambda_{k}}{1-\lambda_{k}}+\frac{\mu_{k}}{1-\mu_{k}}\right)$ where $\mu_{1} \leq \mu_{2}<0<\ldots \leq \lambda_{2} \leq \lambda_{1}$ are the non-zero eigenvalues of $A_{\xi}$. It is also obvious that (ii) implies (iii).

Under the assumption of (iii) it follows from perturbation theory that the eigenspace to a non-zero eigenvalue varies continuously with $p \in M$. In fact, the orthogonal projection onto such an eigenspace can be described as a certain integral over the resolvent of $A_{\xi(p)}$ (cf. Kato [K], p. 178 - 181) and thus depends smoothly on $p$. Hence also the zero-eigenspace as the orthogonal complement of the others varies continuously and is therefore either infinite dimensional or has finite constant dimensions. This proves that (i) follows from (iii).

We are now in a position to compare our definition of isoparametric submanifold with that of Terng [T2]. Terng calls an immersed submanifold $M$ in a separable Hilbert space $V$ proper Fredholm, if the endpoint map $Y:=\exp _{\nu M}: M \rightarrow V$ is Fredholm (i.e. has a Fredholm derivative) and the restriction of $Y$ to each normal disk bundle of finite radius $r$ is proper. While the first condition is a purely local one, and is in fact equivalent to the compactness of the shape operators of $M$, the second condition is a global one. It implies in particular that the immersion itself is proper and hence that $M$ is complete as a metric space. Now, Terng defines an immersed, proper Fredholm submanifold of a Hilbert space of finite codimension to be isoparametric, if $\nu M$ is globally flat (that is has trivial holonomy, not only finite) and if for any parallel normal field $\xi$ the shape operators $A_{\xi(p)}$ and $A_{\xi(q)}$ are orthogonally equivalent for all $p, q \in M$.

Corollary 4.3 Let $M$ be an immersed proper Fredholm submanifold of finite codimension of a separable Hilbert space. Then $M$ is isoparametric (according to our definition) if and only if $M$ is isoparametric in the sense of Terng.

Proof: In Appendix B we show that global flatness of the normal bundle in Terng's definition can be replaced by flatness of $\nu M$. Hence, if $M$ is isoparametric, then it is also isoparametric in the sense of Terng by Theorem 4.2. The other direction also follows immediately from the Theorem except that the regularizability of $M$ is not clear a priori. But this is a consequence of the explicit description of the eigenvalues of $A_{\xi}$ given by Terng [T2], cf. also Lemma 7.5 and its proof. 
For later applications we note the following two results about the regularized trace of compact, self-adjoint operators.

Lemma 4.4 Let $V_{i}, i=1, \ldots, k$, be separable Hilbert spaces and $A_{i}: V_{i} \rightarrow V_{i}$ compact, self adjoint, regularizable operators. If $V:=\bigoplus_{1}^{k} V_{i}$ and $A:=\bigoplus_{1}^{k} A_{i}$, then $A: V \rightarrow V$ is regularizable and $t r_{r} A=\sum_{1}^{k} t r_{r} A_{i}$

Proof: By induction, it suffices to consider the case $k=2$. Let $\mu_{1} \leq \mu_{2} \leq \ldots<$ $0<\ldots \leq \lambda_{2} \leq \lambda_{1}$ and $\mu_{1}^{\prime} \leq \mu_{2}^{\prime} \leq \ldots<0<\ldots \lambda_{2}^{\prime} \leq \lambda_{1}^{\prime}$ be the non-zero eigenvalues, repeated with multiplicities, of $A_{1}$ and $A_{2}$ respectively. For any $N \in \mathbb{N}$ we choose $r, r^{\prime}, s$ and $s^{\prime}$, such that $r+r^{\prime}=s+s^{\prime}=N$ and $\lambda_{1}, \ldots, \lambda_{r}, \lambda_{1}^{\prime}, \ldots, \lambda_{r}^{\prime}$ are the $N$ largest and $\mu_{1}, \ldots, \mu_{s}, \mu_{1}^{\prime}, \ldots, \mu_{s^{\prime}}$ the $N$ smallest eigenvalues of $A$. If none of the sequences $\left\{\mu_{i}\right\},\left\{\mu_{i}^{\prime}\right\},\left\{\lambda_{i}\right\},\left\{\lambda_{i}^{\prime}\right\}$ is finite, then necessarily $r, r^{\prime}, s$ and $s^{\prime}$ tend to infinity together with $N$. From

$\sum_{1}^{s}\left(\lambda_{i}+\mu_{i}\right)+\sum_{1}^{s^{\prime}}\left(\lambda_{i}^{\prime}+\mu_{i}^{\prime}\right) \leq \sum_{1}^{r} \lambda_{i}+\sum_{1}^{r^{\prime}} \lambda_{i}^{\prime}+\sum_{1}^{s} \mu_{i}+\sum_{1}^{s^{\prime}} \mu_{i}^{\prime} \leq \sum_{1}^{r}\left(\lambda_{i}+\mu_{i}\right)+\sum_{1}^{r^{\prime}}\left(\lambda_{i}^{\prime}+\mu_{i}^{\prime}\right)$

the lemma follows, as the left and right hand side of the inequality both tend to $t r_{r} A_{1}+$ $\operatorname{tr}_{r} A_{2}$. If e.g. $\left\{\mu_{i}\right\}$ is finite, say $\mu_{1} \leq \mu_{2} \leq \ldots \leq \mu_{s_{0}}<0$, and the other sequences are not, then $r, r^{\prime}$ and $s^{\prime}$ tend to infinity, $\Sigma \lambda_{i}$ converges and $r \cdot \lambda_{r}$ tends to zero. From $s^{\prime}=N-s_{0} \geq N-r=r^{\prime}$ for large $N$ and $\sum_{1}^{r} \lambda_{i}+\sum_{1}^{r^{\prime}} \lambda_{i}^{\prime}+\sum_{1}^{s_{0}} \mu_{i}+\sum_{1}^{s^{\prime}} \mu_{i}^{\prime}=$ $\sum_{1}^{s_{0}}\left(\lambda_{i}+\mu_{i}\right)+\sum_{s_{0}+1}^{r} \lambda_{i}+\sum_{1}^{s^{\prime}}\left(\lambda_{i}^{\prime}+\mu_{i}^{\prime}\right)-\sum_{r^{\prime}+1}^{s^{\prime}} \lambda_{i}^{\prime}$ the lemma follows also in this case, as $\sum_{r^{\prime}+1}^{s^{\prime}} \lambda_{i}^{\prime} \leq\left(s^{\prime}-r^{\prime}\right) \lambda_{r^{\prime}+1}^{\prime}=\left(r-s_{0}\right) \lambda_{r^{\prime}+1}^{\prime} \leq\left(r-s_{0}\right) \lambda_{r}$ and hence $\sum_{r^{\prime}+1}^{s^{\prime}} \lambda_{i}^{\prime}$ tends to zero. If more than one of the sequences is finite, the lemma is rather obvious.

The final result is essentially due to King and Terng ([KT], Theorem 4.2) who proved it for the $\zeta$-regularization. In fact, our proof is just a simplified version of theirs, adapted to our regularization.

Lemma 4.5 (King and Terng) Let $V$ be a separable Hilbert space and $A, B: V \rightarrow V$ self-adjoint operators with $A$ compact, regularizable and $B$ of finite rank. Then $A+B$ is regularizable and $\operatorname{tr}_{r}(A+B)=\operatorname{tr}_{r} A+\operatorname{tr} B$.

Proof: By induction, we may assume that rank $B=1$ and hence $B(x)=\epsilon\langle x, v\rangle v$ for some $v \in V$ and $\epsilon \in\{ \pm 1\}$. Let $v=\sum_{i} v_{i}$ be a decomposition of $v$ into eigenvectors of 
$A$ to pairwise different eigenvalues. If this sum is finite, the lemma follows immediately, as $V$ then splits orthogonally into $A$ - and $B$ - invariant subspaces $V_{0} \oplus V_{1}$ with $\operatorname{dim} V_{0}<$ $\infty$ and $B\left(V_{1}\right)=0$. If the sum $\sum_{i=1}^{\infty} v_{i}$ is infinite, we let $w_{N}:=\sum_{i=1}^{N} v_{i}$ and $B_{N}(x)=$ $\epsilon<x, w_{N}>w_{N}$. Then $\operatorname{tr}_{r}\left(A+B_{N}\right)=\operatorname{tr} r A+\operatorname{tr} B_{N}$ and $\operatorname{tr} B_{N}=\left\|w_{N}\right\|^{2}$ converges to $\operatorname{tr} B=\|v\|^{2}$ (Note that Lemma 4.4 (b) of $[\mathrm{KT}]$, which is used there to prove $\lim _{N \rightarrow \infty} \operatorname{tr} B_{N}=\operatorname{tr} B$, is not correct). Hence we are left to show that $A+B$ is regularizable and $\lim _{N \rightarrow \infty}\left(\operatorname{tr}_{r} A+B_{N}\right)=\operatorname{tr}_{r}(A+B)$.

Let $\mu_{1} \leq \mu_{2} \leq \ldots<0<\ldots \leq \lambda_{2} \leq \lambda_{1}$ and $\mu_{1}(N) \leq \mu_{2}(N) \leq \ldots<0<\ldots \leq$ $\lambda_{2}(N) \leq \lambda_{1}(N)$ be the non-zero eigenvalues of $A+B$ and $A+B_{N}$, respectively. Then $\mu_{i-1} \leq \mu_{i}\left(A+B_{N}\right) \leq \mu_{i+1}$ and $\lambda_{i+1} \leq \lambda_{i}\left(A+B_{N}\right) \leq \lambda_{i-1}$ for all $N$ and all $i \geq 2$. In fact, this follows from $\lambda_{n+m+1}\left(T_{1}+T_{2}\right) \leq \lambda_{n+1}\left(T_{1}\right)+\lambda_{m+1}\left(T_{2}\right)$ for any compact, self-adjoint operators $T_{1}, T_{2}$ ([DS]) and the fact, that $A+B_{N}$ differs from $A+B$ only by an operator of rank 1 (cf. Lemma 4.5 of $[\mathrm{KT}]$ ). Hence

$$
\lambda_{l}-\lambda_{k+1}+\mu_{k}-\mu_{l+1} \leq \sum_{i=k}^{l}\left(\lambda_{i}+\mu_{i}\right)-\sum_{i=k}^{l}\left(\lambda_{i}(N)+\mu_{i}(N)\right) \leq \lambda_{k}-\lambda_{l+1}+\mu_{l}-\mu_{k-1}
$$

for all $N$ and all $l \geq k \geq 2$. This proves that $A+B$ is regularizable and that for all $\epsilon>0$ there exists $k_{0}$ such that $\left|\sum_{k_{0}}^{\infty}\left(\lambda_{i}+\mu_{i}\right)-\sum_{k_{0}}^{\infty}\left(\lambda_{i}(N)+\mu_{i}(N)\right)\right|<\epsilon / 2$ for all $N$. Since $\lambda_{i}(N) \rightarrow \lambda_{i}$ and $\mu_{i}(N) \rightarrow \mu_{i}$ due to the convergence of $A+B_{N}$ to $A+B$ in norm (cf. [DS]), we finally get $\left|\operatorname{tr}_{r}(A+B)-\operatorname{tr}_{r}\left(A+B_{N}\right)\right|=\left|\sum_{1}^{\infty}\left(\lambda_{i}+\mu_{i}\right)-\sum_{1}^{\infty}\left(\lambda_{i}(N)-\mu_{i}(N)\right)\right|$ for all large $N$.

\section{Lifting isoparametric submanifolds to a Hilbert space}

Isoparametric submanifolds with flat sections of finite dimensional manifolds $X$ can be studied for certain, but important $X$, by lifting them to a Hilbert space and exploiting the theory of isoparametric submanifolds there. This idea, which will be of decisive importance for our restriction theorem, goes back to Terng and Thorbergsson $[\mathrm{TT}]$ in the case of equifocal submanifolds. The goal of this chapter is to describe and simplify their results and to put them into a broader context.

The essential assumption on the finite dimensional ambient manifold $X$ will be the following: $X$ is the base of a Riemannian submersion $\pi: V \rightarrow X$ with minimal fibres, where $V$ denotes (as always) a real separable Hilbert space. The most important example of such a manifold $X$ is a Lie group $G$ with biinvariant metric. In fact, let $V_{G}$ be the set of $H^{1}$-curves $g:[0,1] \rightarrow G$ with $g(0)=e$. Then $V_{G}$ may be identified 
with the Hilbert space $L^{2}([0,1], \mathfrak{g})$ of $L^{2}$-curves in the Lie algebra $\mathfrak{g}$ of $G$ by mapping $g$ onto $u(t):=g^{\prime}(t) g^{-1}(t)$, i.e. onto the curve of tangent vectors pulled back by right translations to the identity. If one considers $V_{G}$ in this way as a Hilbert space (as we will do), then the endpoint map $\pi_{G}: V_{G} \rightarrow G, g \mapsto g(1)$, becomes a Riemannian submersion $([\mathrm{TT}])$. Moreover, $\pi_{G}$ has minimal fibres according to King and Terng $([\mathrm{KT}])$. In the next chapter we will give a simple geometric explanation for this fact.

This example can be extended as follows. If $H \subset G$ is a closed subgroup then the canonical projection $G \rightarrow G / H$ becomes in a natural way a Riemannian submersion with minimal (actually totally geodesic) fibres, too. Therefore also the composition $\pi: V_{G} \rightarrow G \rightarrow G / H$ has this property (the minimality of the fibres follows from Lemma 5.2 below). In particular any compact symmetric space and more generally any normal homogeneous space $X$ is the base of a Riemannian submersion $\pi: V \rightarrow X$ with minimal fibres, where $V$ is a Hilbert space.

If one considers instead of $G / H$ more generally double quotients $G / / H$ where $H \subset G \times G$ operates freely on $G$ by $\left(h_{1}, h_{2}\right) \cdot g=\left(h_{1} g h_{2}^{-1}\right)$ then again $G \rightarrow G / / H$ and hence also the composition $V_{G} \rightarrow G / / H$ is a Riemannian submersion with respect to the natural metric on $G / / H$. But its fibres are in general not minimal.

All of the examples above of Riemannian submersions $\pi: V \rightarrow X$ are of the form $V \rightarrow V / P$ where $P$ is a group of isometries acting freely on $V$ with orbits of finite codimension. The double quotients $G / / H$ arise for example by taking $P:=P(G, H)$ to be the group of $H^{1}$-curves $\quad h:[0,1] \rightarrow G$ with $(h(0), h(1)) \in H$, which acts isometrically on $V_{G}$ by $h . g(t)=h(t) g(t) h^{-1}(0)$. This group has been introduced and studied by Terng in [T3]. We do not know whether there are further examples. In this respect it might be interesting to note that Gromoll and Walchap have recently classified Riemannian submersions $\pi: V \rightarrow X$ with $\operatorname{dim} V<\infty$, that is with $V=\mathbb{R}^{n}$ ([GW1],[GW2]). They show that these are precisely of the above form $V \rightarrow V / P$, where $P$ is a group of glide transformations. Note also that the base $X$ of a Riemannian submersion $\pi: V \rightarrow X$, where $V$ is a Hilbert space, has non-negative curvature by O'Neill's formula and that most known examples of manifolds with non-negative curvature arise in this way (at least up to diffeomorphism).

Let $\pi: V \rightarrow X$ be a Riemannian submersion onto a finite dimensional Riemannian manifold $X$. If $f: M \rightarrow X$ is an isometric immersion we denote by $\hat{f}: \hat{M} \rightarrow V$ its lift to $V$ where $\hat{M}=\{(p, v) \in M \times V \mid f(p)=\pi(v)\}$ and $\hat{f}(p, v)=v$. If $M$ is embedded then $\hat{M}$ can be of course identified with $\pi^{-1}(M)$. Note that $\hat{f}$ is an immersion with the same codimension as $f$ and that the normal spaces of $\hat{f}$ are horizontal. We endow $\hat{M}$ with the metric induced by $\hat{f}$ and observe that the natural projection $\hat{M} \rightarrow M$ then becomes a Riemannian submersion, too. We recall that a differentiable mapping between Hilbert manifolds is called Fredholm if its differentials are Fredholm and that an immersed submanifold $N$ of $V$ is called proper Fredholm if $\exp _{\nu N}: \nu N \rightarrow V$ is Fredholm and the restriction of $\exp _{\nu N}$ to normal vectors of bounded length is proper. It has been shown by Terng in [T2] that $\exp _{\nu N}$ is Fredholm iff the shape operators of 
$N$ are compact.

Lemma 5.1 Let $\pi: V \rightarrow X, f: M \rightarrow X$ and $\hat{f}: \hat{M} \rightarrow V$ be as above. Then:

(i) The normal exponential map $\exp _{\nu \hat{M}}: \nu \hat{M} \rightarrow V$ is Fredholm.

(ii) $\hat{M}$ is proper Fredholm iff $f: M \rightarrow X$ is proper.

\section{Proof:}

(i) If $c:[0,1] \rightarrow X$ is a geodesic segment then the horizontal lifts of $c$ connect the fibres $\pi^{-1}(c(0))$ and $\pi^{-1}(c(1))$ with each other, and therefore induce a diffeomorphism between these fibres. It follows that for any $\hat{\xi}_{0} \in \nu \hat{M}, \exp _{\nu \hat{M}}: \nu \hat{M} \rightarrow V$ maps the submanifold $\left\{\hat{\xi} \in \nu \hat{M} \mid \pi_{*} \hat{\xi}=\pi_{*} \hat{\xi}_{0}\right\}$ diffeomorphically onto the fibre through $\exp \hat{\xi}_{0}$. Since this submanifold and the fibre both have finite codimension in $\nu \hat{M}$ and $\hat{M}$, respectively, the differential of $\exp _{\nu \hat{M}}$ at $\hat{\xi}_{0}$ and hence $\exp _{\nu \hat{M}}$ itself are Fredholm.

(ii) We only prove that " $f$ proper" implies " $\hat{M}$ proper Fredholm", the other direction being almost trivial. Because of (i) it thus suffices to show that any sequence $\hat{\xi}_{n} \in \nu \hat{M}$ with $\left\|\hat{\xi}_{n}\right\| \leq r$ and $\exp \hat{\xi}_{n} \rightarrow v$ has a convergent subsequence. Recall that $\hat{\xi}_{n}$ is a pair $\left(\hat{p}_{n}, \bar{v}_{n}\right)$ where $\hat{p}_{n}=\left(p_{n}, u_{n}\right) \in \hat{M}$ and $\bar{v}_{n} \in T_{\hat{f}\left(\hat{p}_{n}\right)} V$, and thus $\bar{v}_{n}=\left(u_{n}, w_{n}\right)$. Let $\Phi_{t}: T V \rightarrow T V$ be the geodesic flow of $V$, i.e. $\Phi_{t}(u, w)=(u+t w, w)$. Then the vectors $\Phi_{1}\left(\bar{v}_{n}\right)$ are horizontal and of length $\leq r$. By assumption their foot points $\exp \bar{v}_{n}=\exp \hat{\xi}_{n}$ converge to $v$. Hence a subsequence $\Phi_{1}\left(\bar{v}_{n_{k}}\right)$ converges, say to $(v, w)$. Therefore $\bar{v}_{n_{k}}$ converges to $\Phi_{-1}(v, w)$. Since $f\left(p_{n_{k}}\right)=\pi\left(u_{n_{k}}\right)$ converge and $f$ is proper we also may assume that the $p_{n_{k}}$ converge which finally proves the convergence of the $\hat{\xi}_{n_{k}}$.

Remark: A different proof of (i) will follow from Corollary 6.2, which relates the eigenvalues of the shape operators of $\hat{M}$ to the focal points of $M$.

The following result is essentially due to King and Terng who proved it in case $X=G$ a compact Lie group and $V=V_{G}([\mathrm{KT}]$, Theorems 4.12 and 4.17).

Lemma 5.2 Let $\pi: V \rightarrow X, f: M \rightarrow X$ and $\hat{f}: \hat{M} \rightarrow V$ be as above, and assume in addition that $\pi$ has minimal fibres. Then $\hat{M}$ is regularizable. Moreover the regularized mean curvature vector field of $\hat{M}$ is defined and coincides with the horizontal lift of that of $M$.

Proof: Since this result is local we may assume that $M$ is embedded. Let $\hat{p} \in \hat{M}$ and $\hat{\xi} \in \nu_{\hat{p}} \hat{M}$. Then $T_{\hat{p}} \hat{M}$ may be identified with $T_{\hat{p}} F \oplus T_{p} M$ where $F$ is the fibre through $\hat{p}$ and $p=\pi \hat{p}$. The shape operator $A_{\hat{\xi}}^{\hat{M}}$ of $\hat{M}$ therefore decomposes into $A+B+C$ where $A$ and $B$ preserve the decomposition of $T_{\hat{p}} \hat{M}$ with $A\left(T_{p} M\right)=B\left(T_{\hat{p}} \hat{F}\right)=0$, while $C$ interchanges the summands. We may identify $A$ with the shape operator $A_{\hat{\xi}}^{F}$ 
of the fibre and $B$ with the shape operator $A_{\xi}^{M}$ of $M$ in direction $\xi:=\pi_{*} \hat{\xi}$. Since the rank of $B+C$ is at most $2 \cdot \operatorname{dim} T_{p} M$ and hence finite, Lemma 4.5 implies that $A_{\hat{\xi}}^{\hat{M}}$ is regularizable and

$$
\operatorname{tr}_{r} A_{\hat{\xi}}^{\hat{M}}=\operatorname{tr}_{r} A+\operatorname{tr}(B+C)=\operatorname{tr}_{r} A_{\hat{\xi}}^{F}+\operatorname{tr} A_{\xi}^{M}+\operatorname{tr} C=\operatorname{tr} A_{\xi}^{M}
$$

Therefore $\hat{\xi} \rightarrow t r_{r} A_{\hat{\xi}}^{M}$ is linear and we may define the mean curvature vector field $\hat{\eta}$ of $\hat{M}$ by $\left\langle\hat{\eta}, \hat{\xi}>:=\operatorname{tr}_{r} A_{\hat{\xi}}^{\hat{M}}\right.$. Since this latter expression coincides with $\operatorname{tr} A_{\xi}^{M}=<\eta, \xi>$ where $\eta$ denotes the mean curvature vector field of $M, \hat{\eta}$ is the horizontal lift of $\eta$.

As always, we assume that $f: M \rightarrow X$ is an isometric immersion. If for each $p \in M$ and each plane $\sigma \subset \nu_{p} M$ the sectional curvature $K(\sigma)$ of $X$ vanishes we abbreviate this by $K_{X}(\nu M)=0$.

Lemma 5.3 Let $\pi: V \rightarrow X, f: M \rightarrow X$ and $\hat{f}: \hat{M} \rightarrow V$ be as above, $p \in X$ and $U \subset T_{p} X$ a linear subspace.

(i) If $K(\sigma)=0$ for all 2-dimensional subspaces of $U$ then $\exp : U \rightarrow X$ is a totally geodesic isometric immersion. In particular $K_{X}(\nu M)=0$ is equivalent to $M$ having flat sections.

(ii) If $\nu M$ is flat and $K_{X}(\nu M)=0$ then $\nu \hat{M}$ is flat as well. In fact, the horizontal lift of a parallel normal field along an open subset of $M$ is parallel in $\nu \hat{M}$.

\section{Proof:}

(i) Let $\hat{p} \in \pi^{-1}(p)$ and $\hat{U} \subset T_{\hat{p}} V$ be the horizontal lift of $U$ through $\hat{p}$. It suffices to show that the affine subspace $\tilde{U}:=\hat{p}+\hat{U}$ meets fibres always orthogonally and hence projects isometrically into $X$. Let $\hat{q} \in \tilde{U}$ and $v$ be a tangent vector to the fibre at $\hat{q}$. Then there exists a variation of the geodesic $c:[0,1] \rightarrow V$ from $\hat{p}$ to $\hat{q}$ by horizontal geodesics which cover $\pi \circ c$ and whose variational vector field $Y$ satisfies $Y(1)=v$. The orthogonal projection of this Jacobi field $Y$ onto the totally geodesic subspace $\tilde{U}$ is a Jacobi field again. It follows from O'Neill's formula for the curvature of horizontal planes that the O'Neill tensor vanishes on $\hat{U}$ and that therefore $\left\langle Y^{\prime}(0), \hat{w}\right\rangle=-<$ $Y(0), \nabla_{\dot{c}(0)} \hat{W}>=0$ for all $\hat{w} \in \hat{U}$ where $\hat{W}$ is a horizontal extension of $\hat{w}$. Note that $Y(t)$ is vertical. Hence the projection $\bar{Y}$ of $Y$ onto $\tilde{U}$ satisfies $\bar{Y}(0)=\bar{Y}^{\prime}(0)=0$ and thus vanishes identically. In particular $v=Y(1)$ is orthogonal to $\tilde{U}$.

(ii) follows from the vanishing of the O'Neill tensor $O^{\prime} N$ on $\nu \hat{M}$ by O'Neill's curvature formula, cf. chapter 3 .

Theorem 5.4 Let $\pi: V \rightarrow X$ be a Riemannian submersion with minimal fibres from a separable Hilbert space $V$ onto a finite dimensional manifold $X$. Let $M$ be a properly immersed isoparametric submanifold of $X$ with flat sections and $\hat{M}$ its lift to 
$V$. Then $\hat{M}$ is an immersed proper Fredholm isoparametric submanifold of $V$ of finite codimension.

\section{Remarks:}

(i) Under a mild restriction on $X$, also the converse is true: $M$ is isoparametric with flat sections if $\hat{M}$ is isoparametric (Theorem 6.5). (ii) If $X$ is analytic, the minimality assumption on the fibres is not necessary. (Remark 2 following Theorem 6.5).

Proof: By Lemma 5.1, $\hat{M}$ is proper Fredholm, and by Lemma 5.3, $\nu(\hat{M})$ is flat and parallel normal fields along $\hat{M}$ are (at least locally) horizontal lifts of parallel normal fields along $M$. Therefore each (local) parallel manifold $\hat{M}_{\hat{\xi}}$ of $\hat{M}$ is locally the lift of a parallel manifold $M_{\xi}$ of $M$. Let $\Phi_{t}$ and $\hat{\Phi}_{t}$ be the goedesic flows of $X$ and $V$, respectively and $\xi^{*}=\Phi_{1}(\xi), \hat{\xi}^{*}=\hat{\Phi}_{1}(\hat{\xi})$ the corresponding normal fields along $M_{\xi}$ and $\hat{M}_{\hat{\xi}}$. Then $\hat{M}_{\hat{\xi}}$ is regularizable by Lemma 5.2 and $\operatorname{tr} \hat{A}_{\hat{\xi}^{*}}=\operatorname{tr} A_{\xi}$ at corresponding points. Thus $\operatorname{tr} \hat{A}_{\hat{\xi}^{*}}$ is constant as well along $\hat{M}_{\hat{\xi}}$ and $\hat{M}$ is isoparametric.

Isoparametric submanifolds are defined in purely local terms. Therefore one can not expect that they give rise in general to a global foliation of the ambient manifold $X$ by parallel manifolds, even if $X$ is the base of a Riemannian submersion $\pi: V \rightarrow X$ with minimal fibres and the submanifold is embedded. A simple counterexample is furnished by a small geodesic circle on a flat 2-torus. Nevertheless there are many interesting situations where the submanifold defines a global foliation. To study these we make the following definition which extends the notion of a parallel manifold of an isoparametric submanifold with globally flat normal bundle.

Definition Let $X$ be a complete Riemannian manifold and $f: M \rightarrow X$ be an immersed isoparametric submanifold.

(i) If $p \in M$ and $\xi \in \nu_{p} M$ we let $M_{\xi}:=\{\tilde{\xi} \in \nu M \mid \tilde{\xi}$ is obtained from $\xi$ by parallel translation in $\nu M$ along any curve in $M$ starting at $p\}$ and $f_{\xi}: M_{\xi} \rightarrow X$ the restricition of exp to $M_{\xi}$. We call $f_{\xi}\left(M_{\xi}\right)$, or more precisely $f_{\xi}: M_{\xi} \rightarrow X$, a parallel manifold of $M$.

(ii) We say that $M$ defines a global foliation of $X$, if $X$ is the disjoint union of the parallel manifolds of $M$, that is if $X=\bigcup_{\xi} f_{\xi}\left(M_{\xi}\right)$ and if for all $\xi_{1}, \xi_{2} \in \nu M$ either $f_{\xi_{1}}\left(M_{\xi_{1}}\right)=f_{\xi_{2}}\left(M_{2}\right)$ or $f_{\xi_{1}}\left(M_{\xi_{1}}\right) \cap f_{\xi_{2}}\left(M_{\xi_{2}}\right)=\phi$. In this case we call the $f_{\xi}\left(M_{\xi}\right)$ also the leaves of the isoparametric foliation.

\section{Remarks:}

(i) If $f: M \rightarrow X$ defines a global foliation, then $f(M)$ has no self-intersections as locally a neighborhood of $f(M)$ is covered by parallel manifolds. This argument shows more precisely that $f$ factorizes over an injective immersion. Hence $f(M)$ is a closed 
embedded submanifold if in addition $f$ is proper. But even then the parallel manifolds need not be embedded (see below).

(ii) If the isoparametric immersion $f: M \rightarrow X$ is proper, then $X=\cup f_{\xi}\left(M_{\xi}\right)$ is satisfied automatically as each $x \in X$ has a closest point on $f(M)$ and hence lies on a geodesic starting orthogonally from $M$.

Theorem 5.5 Let $X$ be a finite dimensional Riemannian manifold which is the base of a Riemannian submersion $\pi: V \rightarrow X$ with minimal fibres, where $V$ is a separable Hilbert space. Let $M$ be a connected Riemannian manifold and $f: M \rightarrow X$ a properly immersed isoparametric submanifold with flat sections.

(i) If the induced mapping $f_{*}: \pi_{1} M \rightarrow \pi_{1} X$ on the fundamental groups is surjective (in particular if $X$ is simply connected), then $M$ defines a global foliation of $X$.

(ii) If $X$ is simply connected then moreover $f(M)$ has globally flat normal bundle and all leaves of the foliation are closed embedded submanifolds.

Proof: We begin with the proof of (ii) and thus assume that $X$ is simply connected. It follows from the exact homotopy sequence of the fibration $\pi: V \rightarrow X$ that the fibres are connected. Hence also the lift $\hat{M}$ of $M$ to $V$ is connected. By Theorem 5.4 and Corollary 4.3 $\hat{M}$ is isoparametric in the sense of Terng and thus gives rise to an isoparametric foliation of $V$ by closed embedded submanifolds. Moreover the normal bundle of $\tilde{M}:=\hat{f}(\hat{M})$ is globally flat. Since the horizontal lift of a normal vector $\xi \in \nu_{p} M$ to the fibre is parallel in $\nu \tilde{M}$ due to the vanishing of the O'Neill tensor on $\nu \tilde{M}$ and since the fibres are connected, the restriction of a parallel normal field along $\tilde{M}$ to a fibre is the horizontal lift of a normal vector to $M$. Therefore the parallel manifolds of $\tilde{M}$ contain with each point the whole fibre and the foliation of $V$ can be pushed down to a foliation of $X$ by closed embedded submanifolds. These are $f(M)$ and its parallel manifolds since a parallel normal field along $\tilde{M}$ projects down to a parallel normal field along $f(M)$, which also shows that $f(M)$ has globally flat normal bundle.

(i) In the general situation let $\varrho: \tilde{X} \rightarrow X$ be the universal cover of $X$ and $\Gamma$ its group of deck transformations. Furthermore let $f^{*}: M^{*} \rightarrow \tilde{X}$ be the lift of $f$, where $M^{*}=\{(p, \tilde{x}) \in M \times \tilde{X} \mid f(p)=\varrho(\tilde{x})\}$ and $f^{*}(p, \tilde{x})=\tilde{x}$. Then $f^{*}$ is proper as well and we conclude from the surjectivity of $\pi_{1} M \rightarrow \pi_{1} X$ that $M^{*}$ is also connected. Since also $\pi: V \rightarrow X$ can be lifted to $\tilde{X}$, the above results imply that $M^{*}$ defines a foliation of $\tilde{X}$ by closed embedded submanifolds. Since $f^{*}\left(M^{*}\right)$ is invariant under $\Gamma$, each $\gamma \in \Gamma$ permutes the parallel manifolds of $M^{*}$. Hence the projections of these parallel manifolds to $X$ (which are the parallel manifolds of $M$ ) are either disjoint or equal.

\section{Remarks:}

(i) An example for the situation described in (i) of Theorem 5.5 (with $X$ not simply connected) is a totally geodesic $\mathbb{R} P^{n-1} \subset \mathbb{R} P^{n}$. 
(ii) In general the parallel manifolds of $M$ in the Theorem above are not embedded submanifolds (although $f(M)$ is always closed and embedded by the remark before the Theorem). A counterexample is given by $X:=\mathbb{R}^{3} \Gamma$, where $\Gamma$ is the group generated by a glide rotation around the $z$-axis and $M$ is the image of the $z$-axis in $X$. If the angle of rotation is an irrational multiple of $2 \pi$ then the parallel manifolds of $M$, which are geodesics, are neither closed nor embedded submanifolds (except $M$ itself). However, this phenomenon can not occur if $X$ is compact, as we will see below.

Let $f: M \rightarrow X$ be a proper isoparametric immersion with flat sections, where $X$ is the base of a Riemannian submersion $\pi: V \rightarrow X$. By part (i) of Lemma 5.3, the exponential maps exp $: \nu_{p} M \rightarrow X$ are totally geodesic isometric immersions. Thus the sections are globally defined, but again they are neither closed nor embedded submanifolds in general, even if $X$ is a compact simply connected symmetric space. A counterexample is given by the cohomogeneity-1 action on $S^{2} \times S^{2}$ described in section 2. However, we have the following weak compactness result for sections which will be sufficient for later applications.

Theorem 5.6 Let $X$ be a compact Riemannian manifold which is the base of a Riemannian submersion $\pi: V \rightarrow X$ with minimal fibres, where $V$ is a separable Hilbert space. Let $M$ be a connected and properly immersed isoparametric submanifold of $X$ with flat sections which defines a global foliation of $X$. Then there exists for each $p \in M$ a discrete cocompact group $\Lambda$ of isometries of $\nu_{p} M$ such that for any $\xi_{1}, \xi_{2} \in \nu_{p} M, \exp \xi_{1}$ and $\exp \xi_{2}$ lie on the same leaf iff $\xi_{1}$ and $\xi_{2}$ lie on the same $\Lambda$-orbit. In particular, the projection of a section onto the space of leaves factorizes over a compact quotient of the section.

Proof: By the remarks before Theorem 5.5 we may assume that $M$ is a compact embedded submanifold of $X$. We fix $p \in M$ and call $\xi_{1}, \xi_{2} \in \nu_{p} M$ equivalent if $\exp \xi_{1}$ and $\exp \xi_{2}$ lie on the same leaf. We denote by $A$ the equivalence class through $0 \in \nu_{p} M$, i.e. $A=\left\{\xi \in \nu_{p} M \mid \exp \xi \in M\right\}$. Let $\Lambda$ be the group of isometries of $\nu_{p} M$ which preserve the equivalence relation. We first prove that $\Lambda$ acts transitively on $A$. Note that $\exp \left(\nu_{p} M\right)$ meets the parallel manifolds of $M$ always orthogonally as can be seen by lifting the foliation to the Hilbert space $V$. Therefore we may identify the tangent space of $\nu_{p} M$ at $\xi \in A$ with the normal space of $M$ at $\exp \xi$. Now, if $\xi_{1}, \xi_{2} \in A$ and $c$ is any piecewise differentiable curve in $M$ from $\exp \xi_{1}$ to $\exp \xi_{2}$ we let $\lambda: \nu_{p} M \rightarrow \nu_{p} M$ be the affine isometry with $\lambda\left(\xi_{1}\right)=\xi_{2}$ and $d \lambda_{\xi_{1}}=\alpha$ where $\alpha$ denotes the parallel translation along $c$ in $\nu M$. By the very definition of $\lambda$ and the fact that $\exp : \nu_{p} M \rightarrow X$ is a totally geodesic isometric immersion we get that $\exp \left(\xi_{1}+\zeta\right)$ and $\exp \left(\xi_{2}+\alpha(\zeta)\right)$ lie on the same leaf for any $\zeta \in \nu_{p} M$. Thus $\lambda \in \Lambda$ and $\Lambda$ acts transitively on $A$. Moreover the isotropy group of $\Lambda$ at 0 contains the normal holonomy group of $M$ at $p$.

The same arguments can be applied to any equivalence class $A^{\prime}$ containing a regular element. The regular elements are those normal vectors for which the corresponding 
parallel manifold is of highest dimension, and they are dense in $\nu_{p} M$ as one can see again by lifting the foliation to the Hilbert space. Thus $\Lambda$ also acts transitively on $A^{\prime}$. If $\xi \in \nu_{p} M$ there exists $\zeta \in \nu M$ with $\exp \zeta=\exp \xi$ and $\|\zeta\| \leq d:=\operatorname{diam} X$. The parallel translation in $\nu M$ of $\zeta$ to $p$ along a curve in $M$ also has length at most $d$ and is equivalent to $\xi$. For each regular $\xi$ we therefore find a $\lambda \in \Lambda$ with $\|\lambda(\xi)\| \leq d$ and by continuity the same is true for any $\xi \in \nu_{p} M$ provided that we replace $d$ by any $d^{\prime}>d$. Hence $\Lambda$ acts cocompactly on $\nu_{p} M$. It also acts properly discontinuously as there exists, by the compactness of $M$, an $\epsilon>0$ such that $\xi$ is a diffeomorphism on the $\epsilon$-tube in $\nu M$, and thus the $\epsilon$-balls in $\nu_{p} M$ around each $a \in A$ are disjoint. Since the action of $\Lambda$ is proper and the regular elements are dense, it follows finally that $\Lambda$ acts transitively on each equivalence class.

Corollary 5.7 Under the assumptions of the Theorem, the holonomy of $\nu(M)$ is finite and all leaves of the foliation are compact embedded submanifolds.

Proof: In the course of proof of the Theorem it turned out that the normal holonomy group of $M$ at $p$ lies in the isotropy group of $\Lambda$ at $O$. But this is finite as $\Lambda$ acts properly discontinuously on $\nu_{p} M$. Hence $M_{\xi}=\{\tilde{\xi} \in \nu M \mid \tilde{\xi}$ is obtained from $\xi$ by parallel translation in $\nu M$ along curves in $M\}$ is a finite cover of $M$ for each $\xi \in \nu M$. Since we may assume that $M$ is compact it follows that also all leaves are compact and are hence compact embedded submanifolds by the remark preceding Theorem 5.5.

\section{Riemannian submersions and focal points}

The results of this chapter will not be used in the sequel. They rather complement those of the last chapter by proving a converse to Theorem 5.4 and by relating the equifocal submanifolds of Terng-Thorbergsson [TT] to the isoparametric ones.

Our main tool for this is an elementary but basic lemma about Riemannian submersions $\pi: \hat{X} \rightarrow X$ (Lemma 6.1). Essentially it says that focal distances in the direction of a normal vector $\xi$ do not change if one lifts a submanifold from $X$ to $\hat{X}$ (and $\xi$ to $\hat{\xi}$ ). This result was known in special cases ([O'N2], Theorem 4 and [TT], Lemma 5.12) but it does not seem to have been noticed before in this generality. In case $\hat{X}$ is a euclidean space (possibly an infinite dimensional Hilbert space) the lemma immediately relates the eigenvalues of the shape operators of the lifted manifold to focal distances of the submanifold in $X$. In particular the minimality of the fibres of a Riemannian submersion $\pi: V \rightarrow X$, where $V$ is a Hilbert space, is equivalent to a special behaviour of conjugate points in $X$ : For any geodesic $c$ in $X$ the points conjugate to $c(0)$ along $c$ have to lie symmetrically on both sides of $c(0)$, at least in the average. This property is obviously satisfied for symmetric spaces but also more generally for spaces in which geodesics are orbits of 1-parameter groups like normal homogeneous spaces. 
Recall that the differential of the normal exponential map $\exp _{\nu M}: \nu M \rightarrow X$ of a submanifold $M \subset X$ at $\xi \in \nu_{p} M$ may be described by means of $M$-Jacobi fields along $c(t):=\exp t \xi$. These are by definition the variational vector fields of variations of $c$ through geodesics starting perpendicularly from $M$. Alternatively, they are the Jacobi fields $Y$ along $c$ with $Y(0) \in T_{p} M$ and $Y^{\prime}(0)^{T}=-A_{\xi} Y(0)$, where ${ }^{T}$ denotes the tangential component and $A_{\xi}$ the shape operator of $M$ in the direction of $\xi$. If $\mathfrak{J}$ denotes the space of $M$-Jacobi fields along $c(t)=\exp t \xi$ then $T_{\xi} \nu M$ may be identified with $\mathfrak{J}$ by mapping $\dot{\xi}(0)$ onto $Y(t):=\frac{\partial \alpha}{\partial s}(0, t)$, where $\xi(s)$ is a curve in $\nu M$ with $\xi(0)=\xi$ and $\alpha(s, t)=\exp t \xi(s)$. Under this identification, $\left(d \exp _{\nu M}\right)_{\xi}: T_{\xi} \nu M \rightarrow T_{\exp \xi}$ corresponds to $Y \mapsto Y(1)$. This description of $d \exp _{\nu M}$ is also valid in the infinite dimensional case, i.e. if $M$ is a submanifold of a Hilbert manifold. A normal vector $\xi$ is called a multiplicity $-m$ focal direction if $\xi$ lies in the domain of exp and the dimension of the kernel of $d \exp _{\nu M}$ at $\xi$ is equal to $m$.

Lemma 6.1 Let $X$ and $\hat{X}$ be Riemannian manifolds of possibly infinite dimension and $\pi: \hat{X} \rightarrow X$ a Riemannian submersion. Let $M$ be a submanifold of $X$ and $\hat{M}:=$ $\pi^{-1}(M)$. Then $\hat{\xi} \in \nu \hat{M}$ is a multiplicity- $m$ focal direction of $\hat{M}$ if and only if $\pi_{*} \hat{\xi}$ is a multiplicity $-m$ focal direction of $M$. In particular focal distances of $M$ and $\hat{M}$ in corresponding normal directions are equal.

Proof: Let $\xi:=\pi_{*} \hat{\xi}$ and $\mathfrak{J}$ and $\hat{\mathfrak{J}}$ be the spaces of $M$ - and $\hat{M}$-Jacobi fields along $c(t):=\exp t \xi$ and $\hat{c}(t):=\exp t \hat{\xi}$, respectively. We then get the following commutative diagram

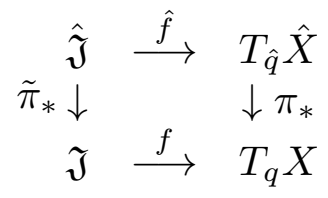

where $\hat{q}=\hat{c}(1), q=c(1), f$ and $\hat{f}$ are the evaluation maps at $t=1$ and $\tilde{\pi}_{*} \hat{Y}(t):=$ $\pi_{*}(\hat{Y}(t))$. We want to show that $\tilde{\pi}_{*}$ induces an isomorphism between the kernels of $\hat{f}$ and $f$, respectively. But this follows by elementary linear algebra provided we know that $\tilde{\pi}_{*}$ is surjective and $\hat{f}$ induces an isomorphism between ker $\tilde{\pi}_{*}=: \hat{\mathfrak{J}}^{v}$ (the space of everywhere vertical $\hat{M}$-Jacobi fields) and ker $\pi_{*}=: \mathfrak{V}_{\hat{q}}$. The first fact is obvious while the second comes essentially from the observation that the horizontal lifts of $c$ define a diffeomorphism between the fibres over $c(0)$ and $c(1)$ (and hence do not focalize). More precisely, if $\tilde{\mathfrak{J}}^{v}$ denotes the set of Jacobi fields along $\hat{c}$ arising from such variations of $\hat{c}$ by horizontal lifts of $c$ then $\tilde{\mathfrak{J}}^{v} \subset \hat{\mathfrak{J}}^{v}$. If $Y \in \hat{\mathfrak{J}}^{v}$ with $Y(0)=0$ then also $Y^{\prime}(0)=0$ as $Y^{\prime}(0)$ is horizontal and $\left.\left\langle Y^{\prime}(0), \eta\right\rangle=-<Y(0), \eta^{\prime}\right\rangle=0$ for any horizontal vector field $\eta$ along $\hat{c}$. The Jacobi fields in $\hat{\mathfrak{J}}^{v}$ are therefore completely determined by their initial value and thus $\tilde{\mathfrak{J}}^{v}=\hat{\mathfrak{J}}^{v}$. Since $\hat{f}: \tilde{\mathfrak{J}}^{v} \rightarrow \mathfrak{V}_{\hat{q}}$ is an isomorphism, the proof of the lemma is completed. 
As in the last chapter we denote by $V$ always a real separable Hilbert space and by $\pi: V \rightarrow X$ a Riemannian submersion onto a finite dimensional manifold. If $N \subset V$ is a submanifold, $\lambda \in \mathbb{R} \backslash\{0\}$, and $\xi \in \nu N$, then $1 / \lambda$ is a multiplicity $-m$ focal direction of $N$ iff $\lambda$ is a multiplicity $-m$ eigenvalue of the shape operator $A_{\xi}$ of $N$ (This is simply because $N$-Jacobi fields in $V$ are of the form $A+t B$ with $A, B$ parallel, $A(0)$ tangential and $\left.B(0)^{T}=-A_{\xi} A(0)\right)$.

Corollary 6.2 Let $\pi: V \rightarrow X$ be a Riemannian submersion, $M \subset X$ a submanifold and $\hat{M}=\pi^{-1}(M)$. Furthermore let $\hat{\xi} \in \nu \hat{M}$ and $\xi:=\pi_{*} \hat{\xi}$. Then $\lambda \in \mathbb{R} \backslash\{0\}$ is a multiplicity $-m$ eigenvalue of the shape operator $A_{\hat{\xi}}$ of $\hat{M}$ iff $1 / \lambda \cdot \xi$ is a multiplicity-m focal direction of $M$.

In case $M$ is a point, the corollary relates the eigenvalues of the shape operator of a fibre to conjugate points in $X$. In particular one gets the following result.

Corollary 6.3 Let $\pi: V \rightarrow X$ be a Riemannian submersion. If the geodesics of $X$ are orbits of 1-parameter groups, in particular if $X$ is a symmetric or more generally a normal homogeneous space, then $\pi$ has minimal fibres.

Proof: Let $c(t):=\varphi_{t}(p)$ where $\varphi_{t}$ is a 1-parameter group of isometries. If $c(t)$ is conjugate to $c(0)$ along $c$ then also $c(t+s)=\varphi_{s}(c(t))$ is conjugate to $c(s)=\varphi_{s}(c(0))$ and of the same order. Choosing $s=-t$ shows that $c(-t)$ is conjugate to $c(0)$. By Corollary 6.2 the eigenvalues of the shape operators of the fibres of $\pi$ therefore come in pairs of opposite sign and thus cancel in the trace.

Another consequence of Corollary 6.2 is the following estimate for the eigenvalues of the shape operator of $\hat{M}$.

Corollary 6.4 Let $\pi: V \rightarrow X$ be a Riemannian submersion with $X$ compact (more generally with positive injectivity radius), $M \subset X$ a submanifold and $\hat{M}=\pi^{-1}(M)$. If $\mu_{1} \leq \mu_{2} \leq \ldots \leq 0 \leq \ldots \leq \lambda_{2} \leq \lambda_{1}$ are the eigenvalues of the shape operator $A_{\hat{\xi}}$ of $\hat{M}$ then there exists a constant $C$ with $\lambda_{k},\left|\mu_{k}\right| \leq C / k$. In particular $\Sigma \lambda_{k}^{s}+\Sigma\left|\mu_{k}\right|^{s}<\infty$ for all $s>1$.

Proof: Let $\xi:=\pi_{*} \hat{\xi}$ and $c(t):=\exp t \xi$. By Corollary 6.2 it suffices to show that the number of focal points (counted with multiplicity) of $M$ along $c$ in $[0, T]$ (and $[-T, 0]$ ) grows at most linearly with $T$. But this number can be estimated by the index of $c_{\left.\right|_{[0, T]}}$ and this in turn by the dimension of broken Jacobi fields along $c_{[0, T]}$ where the distance of break points is less then the injectivity radius of $X$ (cf. $[\mathrm{BC}],[\mathrm{M}]$ ).

In generalization of [TT] we call an immersed submanifold $M$ of $X$ equifocal if $\nu M$ is flat and if focal distances and multiplicities in direction of a normal vector are invariant under parallel translation of this vector in $\nu M$ along any curve. Actually 
Terng and Thorbergsson only considered equifocal manifolds with globally flat and abelian normal bundle in compact symmetric spaces whose metric is induced by the Killing form.

Theorem 6.5 Let $\pi: V \rightarrow X$ be a Riemannian submersion with minimal fibres, $M$ a properly immersed submanifold of $X$ and $\hat{M}$ its lift to $V$. If $X$ admits a compact quotient, then the following statements are equivalent.

(i) $M$ is isoparametric with flat sections,

(ii) $M$ is equifocal with flat sections, and

(iii) $\hat{M}$ is isoparametric.

The equivalence of (ii) and (iii) does not require the minimality of the fibres.

Proof: The implication (i) $\Rightarrow$ (iii) is the content of Theorem 5.4. If $M$ is equifocal with flat sections, then we conclude from Lemma 5.3 and Corollary 6.2 - which do not use the minimality of the fibres - that $\nu \hat{M}$ is flat and that the non-zero eigenvalues of the shape operator $A_{\hat{\xi}}$ of $\hat{M}$ are constant under parallel translation of $\hat{\xi}$. As in the proof of Theorem 4.2 it follows that $\hat{M}$ is isoparametric. Thus (ii) implies (iii) as well. If $\hat{M}$ is isoparametric and $M$ has flat sections then one can reverse the above reasoning to get (i) and (ii) from (iii).

Hence we are left to show that $M$ has flat sections if $\hat{M}$ is isoparametric and $X$ admits a compact quotient. We thus make these two assumptions and by lifting $\pi: V \rightarrow X$ to the universal cover of $X$ we may assume in addition that $X$ is simply connected. We also may assume that $M$ and hence $\hat{M}$ are connected and that $M$ is embedded. It then suffices to show that the horizontal lift of a normal vector to $M$ is a parallel normal field along the fibre since then the O'Neill tensor vanishes on $\nu \hat{M}$ and thus $K_{X}(\nu M)=0$.

Let $W$ be the affine span of $\hat{M}$ in $V$ and $\hat{\xi}_{0} \in \nu \hat{M}$ with $\hat{\xi}_{0}$ tangent to $W$. We denote by $\hat{\xi}$ the normal field along the fibre which is the basic horizontal lift of $\pi_{*} \hat{\xi}_{0}$ and by $\tilde{\xi}$ the parallel normal field along the fibre extending $\hat{\xi}_{0}$. The eigenvalues of $A_{\hat{\xi}}$ and $A_{\tilde{\xi}}$ are both constant because of Corollary 6.2 and since $\hat{M}$ is isoparametric. But these eigenvalues are given by $\left\langle\hat{\xi}, n_{i}>\right.$ and $<\tilde{\xi}, n_{i}>$, respectively, where the $n_{i}$ are the curvature normals. Hence $\hat{\xi}-\tilde{\xi}$ is perpendicular to the $n_{i}$ and therefore also to $W$. Since $\tilde{\xi}$ is always tangent to $W$ and $\hat{\xi}=\tilde{\xi}+(\hat{\xi}-\tilde{\xi})$ has the same length as $\tilde{\xi}$, $\hat{\xi}$ and $\tilde{\xi}$ necessarily coincide. The parallel manifold $\hat{M}_{\tilde{\xi}}$ contains therefore in particular with each point the whole fibre through that point and the same is true for $W$ which is a union of parallel manifolds.

It follows from the Cheeger-Gromoll splitting theorem that $X$ splits as $X_{1} \times \mathbb{R}^{k}$ with compact $X_{1}$. Since $X_{1} \times\{x\}$ has no focal points in $X$ and $X$ is simply connected, the inverse images $\pi^{-1}\left(X_{1} \times\{x\}\right)$ are connected totally geodesic submanifolds and hence parallel affine subspaces. Thus we may assume that $V=V_{1} \times \mathbb{R}^{k}$ and $\pi\left(\left(v_{1}, x\right)\right)=$ $\left(\bar{\pi} v_{1}, x\right)$, where $\bar{\pi}: V_{1} \rightarrow X_{1}$. The intersection of $W$ (the affine span of $\left.\hat{M}\right)$ with $V_{1} \times\{x\}$ 
is either empty or $V_{1} \times\{x\}$. In fact $W \cap\left(V_{1} \times\{x\}\right)$ is an affine subspace which contains with each point the whole fibre of $\pi$ and due to the compactness of $X_{1}$ these fibres span $V_{1} \times\{x\}$ as an affine subspace. Hence $W$ is of the form $W=V_{1} \times U$ where $U$ is an affine subspace of $\mathbb{R}^{k}$. But this immediately implies that also the parallel translation of a $\hat{\xi}_{0} \in \nu \hat{M}$, which is perpendicular to $W$, coincides with the basic horizontal lift of $\pi_{*} \hat{\xi}_{0}$.

\section{Remarks:}

1. Without the compactness assumption on $X$, (iii) does not imply (i) or (ii) in the Theorem. A counterexample is given by taking $V$ to be $\mathbb{R}^{3}, X$ the quotient of $\mathbb{R}^{3}$ by a 1-parameter family of glide rotations around an axis $A$ and $M$ the point in $X$ corresponding to $A$. Then $\hat{M}$ is an isoparametric submanifold but $M$ does not have flat sections.

2. In an analytic Riemannian manifold isoparametric submanifolds are always equifocal. In fact, the mean curvature of the parallel manifolds is given along $\exp t \xi$ as the $\operatorname{logarithmic}$ derivative of the volume distortion $v(t):=\left|\operatorname{det} d \tilde{e x p}_{t \xi}\right|$, where ex̃p denotes the normal exponential map. If this logarithmic derivative is invariant under parallel translation of $\xi$ for small $t$ then $v(t)$ itself is invariant under parallel translation of $\xi$ for small $t$ and hence in the analytic case for all $t$. But the zeros of $v(t)$ are precisely the focal distances. Hence (i) implies (iii) without the minimality assumption on the fibres, if $X$ is analytic.

\section{A Chevalley-type restriction theorem}

Let $X$ be a compact Riemannian manifold which is the base of a Riemannian submersion $\pi: V \rightarrow X$ with minimal fibres, where $V$ is a separable Hilbert space, and let $M$ be a properly immersed isoparametric submanifold of $X$ with flat sections which defines a global foliation of $X$. (A typical example is $X$ a compact Lie group with biinvariant metric and $M$ the conjugacy class of a regular element). Furthermore let $p \in M$ and $\Sigma:=\exp \left(\nu_{p} M\right)$ a section (which in general will be only the image of a totally geodesic isometric immersion, see Lemma 5.3). For any space $A$ of functions on $X$ or $\Sigma$ we denote by $A^{M}$ the subspace of functions which are constant on the leaves of the foliation (the intersection of the leaves with $\Sigma$, respectively). Finally we denote for any Riemannian manifold $N$ by $C^{\Delta}(N)$ the subspace of $C^{\infty}$-functions which consists finite sums of eigenfunctions of the Laplacian. For example, $C^{\Delta}\left(S^{n}\right)$ is the space of restrictions of polynomials on $\mathbb{R}^{n+1}$ to $S^{n}$, and is hence a finitely generated algebra. In case of the isometrically immersed $\Sigma$ above we interpret $C^{\Delta}(\Sigma)$ as $\{f: \Sigma \rightarrow \mathbb{R} \mid$ $\left.f \circ \exp _{\left.\right|_{\nu_{p} M}} \in C^{\Delta}\left(\nu_{p} M\right)\right\}$, which of course coincides with the space of finite sums of eigenfunctions of the Laplacian on $\Sigma$, if $\Sigma$ is embedded.

Remark: If $G$ is a group of isometries of the Riemannian manifold $N$, then a function 
$f \in C^{\infty}(N)$ is called $G$-finite if the vector space spanned by the functions $f \circ g, g \in G$, is finite dimensional. If $G$ is in addition compact and transitive, then it is not hard to see that $C^{\Delta}(N)$ coincides with the space of $G$-finite functions on $N$. If moreover $N$ is $G$-equivariantly embedded into some euclidean space (which is always possible by a result of D. Moore) then it has been shown by Helgason [Hel1] that the space of $G$-finite functions and hence $C^{\Delta}(N)$ coincides with the space of restrictions of polynomials from euclidean space to $N$, just as in the case of the sphere.

A major goal of this section is the proof of the following Chevalley-type restriction theorem.

Theorem 7.1 Under the assumptions above $C^{\Delta}(X)^{M}$ and $C^{\Delta}(\Sigma)^{M}$ are isomorphic. In fact, an isomorphism is given by restricting each $f \in C^{\Delta}(X)^{M}$ to $\Sigma$.

Note that we may identify $C^{\Delta}(\Sigma)^{M}$ with $C^{\Delta}\left(\nu_{p} M\right)^{\Lambda}$ where $\Lambda$ is the discrete cocompact group of isometries of $\nu_{p} M$ introduced in Theorem 5.6. Hence $C^{\Delta}(\Sigma)^{M}$ may be viewed as the space of finite sums of eigenfunctions on a flat torus which are invariant under a finite group of isometries, namely the quotient of $\Lambda$ by a lattice.

To prepare the proof of the theorem we begin with several lemmas. Let $\Gamma \subset \mathbb{R}^{n}$ be a lattice. Then $C^{\Delta}\left(\mathbb{R}^{n}\right)^{\Gamma}=\left\{\sum_{\omega \in \Gamma^{*}} a_{\omega} e^{2 \pi i \omega} \mid a_{\omega} \in \mathbb{C}, a_{-\omega}=\bar{a}_{\omega}, a_{\omega}=0\right.$ except for finitely many $\omega\}$, where $\Gamma^{*}:=\left\{\omega: \mathbb{R}^{n} \rightarrow \mathbb{R} \mid \omega\right.$ linear, $\left.\omega(\Gamma) \subset \mathbb{Z}\right\}$ denotes the dual lattice. If $f=\sum_{\omega \in \Gamma^{*}} a_{\omega} e^{2 \pi i \omega}$, we call $\operatorname{supp}_{\Gamma}(f):=\left\{\omega \in \Gamma^{*} \mid a_{\omega} \neq 0\right\}$ the $\Gamma$-support of $f$. The following lemma will be crucial.

Lemma 7.2 Let $\alpha \in \Gamma^{*}$ and $f \in C^{\Delta}\left(\mathbb{R}^{n}\right)^{\Gamma}$. If $f \tan \pi \alpha$ or $f \cot \pi \alpha$ extends to a $C^{\infty}$ function $g$ on $\mathbb{R}^{n}$, then $g \in C^{\Delta}\left(\mathbb{R}^{n}\right)^{\Gamma}$ and $\operatorname{supp}_{\Gamma}(g)$ is contained in the convex hull of $\operatorname{supp}_{\Gamma}(f)$.

Proof: We only consider $g=f \cot \pi \alpha$, the other case being similar. Let $f=\Sigma a_{\omega} e^{2 \pi i \omega}$. Since $g$ is $\Gamma$-invariant, we may expand it also into a Fourier series $g=\sum_{\omega \in \Gamma^{*}} b_{\omega} e^{2 \pi i \omega}$. From

$$
\left(e^{2 \pi i \alpha}+1\right) \Sigma a_{\omega} e^{2 \pi i \omega}=\left(e^{2 \pi i \alpha}-1\right) \Sigma b_{\omega} e^{2 \pi i \omega}
$$

we get $a_{\omega-\alpha}+a_{\omega}=b_{\omega-\alpha}-b_{\omega}$ and hence

$$
b_{\omega-\alpha}=b_{\omega}+a_{\omega-\alpha}+a_{\omega}
$$

or equivalently

$$
b_{\omega+\alpha}=b_{\omega}-\left(a_{\omega}+a_{\omega+\alpha}\right)
$$

for all $\omega \in \Gamma^{*}$. 
If $\omega_{0}$ is not contained in the convex hull of $\operatorname{supp}_{\Gamma}(f)$ then this is also the case either for all $\omega_{0}+k \alpha$ or for all $\omega_{0}-k \alpha$ with $k \in \mathbb{N}_{0}$. In the first case we conclude from $(* *) \quad b_{\omega_{0}}=b_{\omega_{0}+\alpha}=b_{\omega_{0}+2 \alpha}=\ldots$ and hence $b_{\omega_{0}}=0$, since $g$ lies in $L^{2}$ when considered as a function on $\mathbb{R}^{n} / \Gamma$. Similarly we conclude in the second case from $(*) \quad b_{\omega_{0}}=0$, which completes the proof.

Lemma 7.3 Let $\Lambda \subset I\left(\mathbb{R}^{k}\right)$ be a discrete cocompact group of isometries of $\mathbb{R}^{k}$ and $W \subset \Lambda$ a subgroup generated by reflections in a $\Lambda$-invariant family $\mathfrak{H}$ of affine hyperplanes. Then $W$ does not split off a finite factor and is thus an affine Weyl group (whose action on $\mathbb{R}^{k}$, however, might contain a trivial summand). In particular each $H \in \mathfrak{H}$ is contained in an infinite family of parallel affine hyperplanes of $\mathfrak{H}$.

Proof: From the theory of affine reflection groups we have (after a possible adjustment of the origin) a decomposition of $\mathbb{R}^{k}$ into $V_{0} \oplus V_{1} \oplus V_{2}$ and of $W$ into $W_{1} \times W_{2}$ where $W_{1}$ is an affine Weylgroup acting only on $V_{1}, W_{2}$ is a finite linear reflection group acting only on $V_{2}$ and $V_{0}$ is the set of fixed vectors of $W$. The reflection hyperplanes of $W_{2}$ are precisely those which have no further parallel hyperplanes in $\mathfrak{H}$. This subset of hyperplanes is left invariant by $\Lambda$ and hence also their intersection, which is equal to $V_{0} \oplus V_{1}$. Since $\Lambda$ acts cocompactly, $V_{0} \oplus V_{1}=\mathbb{R}^{k}$ and thus $W_{2}=\{e\}$.

Lemma 7.4 Let $\Lambda \subset I\left(\mathbb{R}^{k}\right)$ be a discrete cocompact group of isometries of $\mathbb{R}^{k}$. Then $C^{\Delta}\left(\mathbb{R}^{k}\right)^{\Lambda}$ is dense in $C^{\infty}\left(\mathbb{R}^{k}\right)^{\Lambda}$ with respect to the supremum norm.

Proof: Let $\Gamma \subset \Lambda$ be the subgroup of translations in $\Lambda$. Then $\Gamma$ is a lattice and $\bar{\Lambda}:=\Lambda / \Gamma$ is a finite group which operates on $\mathbb{R}^{k} / \Gamma$. Since $\mathbb{R}^{k} / \Gamma$ is compact, $C^{\Delta}\left(\mathbb{R}^{k} / \Gamma\right)$ is dense in $C^{\infty}\left(\mathbb{R}^{k} / \Gamma\right)$ with respect to the supremum norm. Hence also $C^{\Delta}\left(\mathbb{R}^{k} / \Gamma\right)^{\Lambda}$ is dense in $C^{\infty}\left(\mathbb{R}^{k} / \Gamma\right)^{\bar{\Lambda}}$ as follows by averaging an approximating sequence in $C^{\Delta}\left(\mathbb{R}^{k} / \Gamma\right)$ over $\bar{\Lambda}$. From this the lemma follows immediately.

Our final preparation for the proof of the theorem is an extension of remark 5.1 of $[\mathrm{KT}]$ in which the mean curvature vector field of an isoparametric hypersurface in a Hilbert space had been given.

Let $\hat{M} \subset V$ be a connected, proper Fredholm isoparametric submanifold of finite codimension of a separable Hilbert space $V, p \in \hat{M}$ and $\hat{\Sigma}:=p+\nu_{p} \hat{M}$ be the affine normal space at $p$. Let $\mathfrak{H}$ denote the set of focal hyperplanes of $\hat{M}$ in $\hat{\Sigma}, \hat{\Sigma}_{\text {reg }}:=$ $\hat{\Sigma} \backslash \bigcup_{H \in \mathfrak{H}} H$ the set of regular points and $W$ the group generated by reflections in the hyperplanes of $\mathfrak{H}$. To each $H \in \mathfrak{H}$ there is associated its so called multiplicity $m_{H}$ which is equal to the dimension of the curvature sphere of $\hat{M}$ through $p$ focalizing on $H$. This number is invariant under $W$, that is $m_{w(H)}=m_{H}$ for all $w \in W$, and it is also independent of $p \in \hat{\Sigma}_{\text {reg }}$. More precisely, for any $q \in \hat{\Sigma}_{\text {reg }}$ the affine normal space of the parallel manifold $\hat{M}_{\xi}$ of $\hat{M}$ through $q$ is equal to $\hat{\Sigma}$ and the focal hyperplanes of 
$\hat{M}_{\xi}$ in that affine normal space and their multiplicities coincide with that of $M$. It is well known that $\mathfrak{H}$ contains finitely many hyperplanes, say $H_{1}, \ldots, H_{r}$, such that each $H \in \mathfrak{H}$ is parallel to one of these and the $H_{i}$ have a point in common. We endow $\hat{\Sigma}$ with the structure of a euclidean vector space by choosing as origin a point from the intersection of the $H_{i}$. Let $v_{i} \in \hat{\Sigma}$ be a unit vector perpendicular to $H_{i}$. We assume that $\mathfrak{H}$ contains with each $H \in \mathfrak{H}$ another parallel hyperplane and let $l_{i}>0$ be the common distance between neighboring parallel hyperplanes in the infinite family determined by $H_{i}$. Since the reflection in a hyperplane interchanges its parallel neighbors there are numbers $m_{i}^{+}, m_{i}^{-}, i \in\{1, \ldots, r\}$, such that $m_{H}=m_{i}^{+}$if $H=2 k l_{i} v_{i}+H_{i}$ and $m_{H}=m_{i}^{-}$ if $H=(2 k+1) l_{i} v_{i}+H_{i}$ for all $k \in \mathbb{Z}$.

Lemma 7.5 The parallel manifolds of $\hat{M}$ through regular points of $\hat{\Sigma}$ are regularizable. More precisely, the shape operator of the parallel manifold of $\hat{M}$ through $q \in \hat{\Sigma}_{r e g}$ in direction $\xi \in \hat{\Sigma}$ is equal to $\langle\hat{\eta}(q), \xi>$ where $\hat{\eta}$ is given by

$$
\hat{\eta}=\sum_{i=1}^{r} \frac{\pi}{2 l_{i}}\left(m_{i}^{-} \tan \pi \alpha_{i}-m_{i}^{+} \cot \pi \alpha_{i}\right) v_{i}
$$

and $\alpha_{i}$ is the linear functional on $\hat{\Sigma}$ with $\alpha_{i}(x)=<x, v_{i}>/ 2 l_{i}$. Note that $2 \alpha_{i}(x) \in \mathbb{Z}$ iff $x$ lies on a focal hyperplane parallel to $H_{i}$, which is in particular the case if $x+\mathfrak{H} \subset \mathfrak{H}$.

Proof: The non-zero eigenvalues of the shape operator in the direction of $\xi$ are the numbers $1 / t$ with $q+t \xi \in \bigcup H$ and their multiplicity is the sum over all $m_{H}$ for which $H \in \mathfrak{H}$

$q+t \xi \in H$. These eigenvalues fall naturally into $2 r$ classes, namely into the classes of eigenvalues $1 / t$ with multiplicity $m_{i}^{+}$where $q+t \xi \in 2 k l_{i} v_{i}+H_{i}$, i.e. $\left\langle q+t \xi, v_{i}\right\rangle=2 k l_{i}$ or equivalently $1 / t=\frac{\left\langle\xi, v_{i}>\right.}{2 k l_{i}-<q, v_{i}>}$ and the classes of eigenvalues $1 / t$ with multiplicity $m_{i}^{-}$where $q+t \xi \in(2 k+1) l_{i} v_{i}+H_{i}$, i.e. $\frac{1}{t}=\frac{<\xi, v_{i}>}{(2 k+1) l_{i}-<q, v_{i}>}, k \in \mathbb{Z}$.

The regularized trace for these subclasses exists, since $\sum_{k \in \mathbb{Z}} \frac{1}{z+k}:=\frac{1}{z}+\sum_{k=1}^{\infty}\left(\frac{1}{z+k}+\right.$ $\left.\frac{1}{z-k}\right)=\pi \cot \pi z$ by the well known partial fraction formula for the cotangent. More precisely $m_{i}^{+} \cdot \sum_{k \in \mathbb{Z}} \frac{<\xi, v_{i}>}{2 k l_{i}-<q, v_{i}>}=-\frac{m_{i}^{+}<\xi, v_{i}>}{2 l_{i}} \pi \cot \pi \alpha_{i}(q)$ and $m_{i}^{-} \cdot \sum_{k \in \mathbb{Z}} \frac{<\xi, v_{i}>}{(2 k+1) l_{i}-<q, v_{i}>}=$ $m_{i}^{-} \frac{\leq \xi, v_{i}>}{2 l_{i}} \pi \tan \pi \alpha_{i}(q)$, where $\alpha_{i}(q)=\frac{\leq q, v_{i}>}{2 l_{i}}$. Thus the regularized trace of the shape operator exists by Lemma 4.4 and is equal to the stated formula.

Proof of Theorem 7.1: We may assume that $M$ is an embedded submanifold of $X$. Let $\hat{M}:=\pi^{-1} M$ and $\hat{M}_{0}$ be a connected component of $\hat{M}$. Then $\hat{M}_{0}$ is a proper Fredholm isoparametric submanifold of $V$ of finite codimension and $\hat{M}$ consists of certain parallel manifolds of $\hat{M}_{0}$ which are also regular, i.e. have the same codimension as $\hat{M}_{0}$. Let $\hat{p} \in \hat{M}_{0}$ and $\hat{\Sigma}:=\hat{p}+\nu_{\hat{p}} \hat{M}_{0}$ be the affine normal space. According to Terng [T2], the restriction mapping defines an isomorphism from $C^{\infty}(V)^{\hat{M}_{0}}$ 
onto $C^{\infty}(\hat{\Sigma})^{\hat{M}_{0}}$ (the set of $C^{\infty}$-function on $\hat{\Sigma}$ which are constant on the intersections of $\hat{\Sigma}$ with the parallel manifolds of $\left.\hat{M}_{0}\right)$ and $C^{\infty}(\hat{\Sigma})^{\hat{M}_{0}}=C^{\infty}(\hat{\Sigma})^{W}$ where $W$ is the group generated by reflections in the focal hyperplanes of $\hat{M}_{0}$ in $\hat{\Sigma}$. This isomorphism yields an isomorphism $C^{\infty}(V)^{\hat{M}} \rightarrow C^{\infty}(\hat{\Sigma})^{\hat{M}}$ where $C^{\infty}(V)^{\hat{M}}$ is the set of $C^{\infty}$-functions on $V$, which are constant on the preimages under $\pi$ of the parallel manifolds of $M$ in $X$ and $C^{\infty}(\hat{\Sigma})^{\hat{M}}$ is the set of their restrictions to $\hat{\Sigma}$. Since we may identify $C^{\infty}(X)^{M}$ with $C^{\infty}(V)^{\hat{M}}$ and $C^{\infty}(\Sigma)^{M}$ with $C^{\infty}(\hat{\Sigma})^{\hat{M}}$, by mapping $f$ to $f \circ \pi$, the restriction mapping $C^{\infty}(X)^{M} \rightarrow C^{\infty}(\Sigma)^{M}$ is an isomorphism, where $C^{\infty}(\Sigma):=\left\{f: \Sigma \rightarrow \mathbb{R} \mid f \circ \exp \in C^{\infty}\left(\nu_{p} M\right)\right\}=\left\{f: \Sigma \rightarrow \mathbb{R} \mid f \circ \pi \in C^{\infty}(\hat{\Sigma})\right\}$. Note that we may identify $\hat{\Sigma}$ with $\nu_{p} M$ by means of the isometry $\hat{p}+\hat{\xi} \mapsto \pi_{*}(\hat{\xi})$ and that $\pi: \hat{\Sigma} \rightarrow X$ corresponds to exp $: \nu_{p} M \rightarrow X$ under this identification. Let $\Lambda$ be the group of isometries of $\hat{\Sigma}$ which map each $x \in \hat{\Sigma}$ to an equivalent point, i.e. to a point $y$ such that $\pi x$ and $\pi y$ lie on the same parallel manifold of $M$. Then $C^{\infty}(\hat{\Sigma})^{\hat{M}}=C^{\infty}(\hat{\Sigma})^{\Lambda}$ by Theorem 5.6 and the action of $\Lambda$ on $\hat{\Sigma}$ is discrete and cocompact. Moreover $\Lambda$ leaves the set $\mathfrak{H}$ of focal hyperplanes of $\hat{M}_{0}$ invariant and contains $W$. Hence $W$ is an affine Weyl group by Lemma 7.3 and each $H \in \mathfrak{H}$ belongs to an infinite family of parallel focal planes.

Let $\delta: C^{\infty}(\Sigma)^{M} \rightarrow C^{\infty}(\Sigma)^{M}$ be defined by $\delta(f):=\left(\Delta^{X} \tilde{f}\right)_{\left.\right|_{\Sigma}}$ where $\Delta^{X}$ denotes the Laplacian of $X$ and $\tilde{f} \in C^{\infty}(X)^{M}$ the extension of $f$ to $X$ along the parallel manifolds of $M$. Note that $\Delta^{X}$ leaves $C^{\infty}(X)^{M}$ invariant. In fact, the projection from $X$ onto the space of leaves of the isoparametric foliation is a Riemannian submersion outside the singular leaves, and the mean curvature vector field of the leaves is a basic horizontal vector field. If $f \in C^{\infty}(X)^{M}, \Delta^{X} f$ is therefore constant along the regular leaves, by the standard formula for the Laplacians of a Riemannian submersion and hence constant on all leaves by continuity.

Let $N_{1}, N_{2} \subset X$ be two submanifolds of $X$ of complementary dimension which meet orthogonally at $q$. Then

$$
\Delta^{X} f(q)=\Delta^{N_{1}} f_{\left.\right|_{N_{1}}}(q)+\Delta^{N_{2}} f_{\left.\right|_{N_{2}}}(q)+\eta_{1}(f)+\eta_{2}(f)
$$

where $\eta_{1}$ and $\eta_{2}$ denote the mean curvature vectors of $N_{1}$ and $N_{2}$ at $q$. This formula follows easily from $\Delta^{X} f(q)=-\operatorname{tr} \operatorname{Hess}_{q} f=-\sum_{i}<\nabla_{e_{i}}$ gradf, $e_{i}>$ by using an orthonormal basis $\left\{e_{i}\right\}$ of $T_{q} X$ compatible with the decomposition $T_{q} N_{1} \oplus T_{q} N_{2}$. Specializing it to $N_{1}=\Sigma$ (which we may assume to be embedded for this consideration) and $N_{2}$ a parallel manifold $M_{\xi}$ of $M$ of maximal dimension yields

$$
\delta(f)(q)=\Delta^{\Sigma} f(q)+\eta(f)
$$

for all $f \in C^{\infty}(\Sigma)^{M}$, where $q \in \Sigma \cap M_{\xi}$ and $\eta$ denotes the mean curvature of $M_{\xi}$ at $q$.

We now identify $C^{\infty}(\Sigma)^{M}$ with $C^{\infty}(\hat{\Sigma})^{\Lambda}$ by means of $f \mapsto f \circ \pi_{\left.\right|_{\hat{\Sigma}}}$ and denote the endomorphism of $C^{\infty}(\hat{\Sigma})^{\Lambda}$ corresponding to $\delta$ by $\hat{\delta}$. Since the regularized mean 
curvature $\hat{\eta}$ of the isoparametric submanifold $\pi^{-1}\left(M_{\xi}\right)$ at $\hat{q} \in \hat{\Sigma} \cap \pi^{-1}(q)$ corresponds under $\pi_{*}$ to $\eta$ by Lemma 5.2, we get

$$
\hat{\delta} f(\hat{q})=\Delta^{\hat{\Sigma}} f(\hat{q})+\hat{\eta}(f)
$$

for all $f \in C^{\infty}(\hat{\Sigma})^{\Lambda}$. But for $\hat{\eta}$ we have the explicit formula of Lemma 7.5 and thus obtain (using the notation introduced before Lemma 7.5)

$$
\hat{\delta}(f)=\Delta^{\hat{\Sigma}}(f)+\sum_{j=1}^{r} \frac{\pi}{2 l_{j}}\left(m_{j}^{-} \tan \pi \alpha_{j}-m_{j}^{+} \cot \pi \alpha_{j}\right) v_{j}(f)
$$

for all $f \in C^{\infty}(\hat{\Sigma})^{\Lambda}$. Recall that $\hat{\Sigma}$ is viewed as a vector space by introducing as origin a point through which passes a focal hyperplane from each infinite family of parallel focal hyperplanes and that the $\alpha_{j}$ are linear functionals on $\hat{\Sigma}$.

Our aim is to prove that the isomorphism $C^{\infty}(X)^{M} \rightarrow C^{\infty}(\hat{\Sigma})^{\Lambda}, f \mapsto f \circ \pi_{\left.\right|_{\hat{\Sigma}}}$, maps $C^{\Delta}(X)^{M}$ onto $C^{\Delta}(\hat{\Sigma})^{\Lambda}$. Denoting the inverse mapping by $\psi$, the essential step will be to show that $\psi\left(C^{\Delta}(\hat{\Sigma})^{\Lambda}\right) \subset C^{\Delta}(X)^{M}$ or equivalently that each $f \in C^{\Delta}(\hat{\Sigma})^{\Lambda}$ is a finite sum of eigenfunctions of $\hat{\delta}=\psi^{-1} \circ \Delta^{X} \circ \psi$. This in turn will follow, if each such $f$ lies in a finite dimensional $\hat{\delta}$-invariant subspace on which $\hat{\delta}$ can be diagonalized. But the last condition is automatic as $\hat{\delta}$ is symmetric on $C^{\infty}(\hat{\Sigma})^{\Lambda}$ with respect to the inner product $<f, g\rangle:=<\psi(f), \psi(g)>_{L^{2}(X)}$.

Let $\Gamma$ be the lattice of translations of $\Lambda$ and $\Gamma_{2}:=2 \Gamma$. Then $\alpha_{j} \in \Gamma_{2}^{*}$ by Lemma 7.5. Hence $v_{j}(f) \tan \pi \alpha_{j}$ as well as $v_{j}(f) \cot \pi \alpha_{j}$ are $\Gamma_{2}$-invariant functions. They are smooth in the whole of $\hat{\Sigma}$ as $\tan \pi \alpha_{j}$ and $\cot \pi \alpha_{j}$ only have poles of first order which moreover are contained in the focal hyperplanes $k l_{j} v_{j}+H_{j}, k \in \mathbb{Z}$, and $v_{j}(f)$ vanishes there due to the invariance of $f$ under reflections along these hyperplanes. Each $f \in C^{\Delta}(\hat{\Sigma})^{\Lambda}$ is $\Gamma_{2}$-invariant and can thus be developed into a Fourier series $\sum_{\omega \in \Gamma_{2}^{*}} a_{\omega} e^{2 \pi i \omega}$ which is finite by assumption. Since $e^{2 \pi i \omega}$ is an eigenfunction of $\Delta^{\hat{\Sigma}}$ and $v_{j}$, we conclude from Lemma 7.2 and the above formula for $\hat{\delta}$ that $\hat{\delta}$ leaves $C^{\Delta}(\hat{\Sigma})^{\Lambda}$ invariant and that moreover the $\Gamma_{2}$-support of $\hat{\delta} f$ is contained in the convex hull of that of $f$. This shows in particular that $\hat{\delta}$ leaves invariant the finite dimensional subspaces $\left\{f \in C^{\Delta}(\hat{\Sigma})^{\Lambda} \mid \operatorname{supp}_{\Gamma_{2}}(f) \subset B_{r}(0)\right\}, r>0$, which fill out completely $C^{\Delta}(\hat{\Sigma})^{\Lambda}$. By the remarks above this implies $\psi\left(C^{\Delta}(\hat{\Sigma})^{\Lambda}\right) \subset C^{\Delta}(X)^{M}$.

Equality follows now by a standard density argument. From Lemma 7.4 we get that $C^{\Delta}(\hat{\Sigma})^{\Lambda}$ is dense in $C^{\infty}(\hat{\Sigma})^{\Lambda}$ with respect to the supremum norm and hence also that $\psi\left(C^{\Delta}(\hat{\Sigma})^{\Lambda}\right)$ is dense in $C^{\infty}(X)^{M}$ and thus in $C^{\Delta}(X)^{M}$. Since $C^{\Delta}(X)$ is the algebraic direct sum of the eigenspaces $E_{k}, k \in \mathbb{N}$, of $\Delta^{X}$ and since $\Delta^{X}$ leaves $C^{\Delta}(X)^{M}$ invariant, we have $C^{\Delta}(X)^{M}=\bigoplus_{k} E_{k}^{M}$ (algebraic direct sum) with $E_{k}^{M}=E_{k} \cap C^{\infty}(X)^{M}$. From $\Delta^{X} \circ \psi=\psi \circ \hat{\delta}$ it follows that $\Delta^{X}$ leaves $\psi\left(C^{\Delta}(\hat{\Sigma})^{\Lambda}\right)$ invariant too, and hence that 
$\psi\left(C^{\Delta}(\hat{\Sigma})^{\Lambda}\right)=\bigoplus_{k} \bar{E}_{k}^{M}$ for certain $\bar{E}_{k}^{M} \subset E_{k}^{M}$. If $\bar{E}_{k}^{M} \neq E_{k}^{M}$ for some $k$, there would exist an $f \in C^{\Delta}(X)^{M} \backslash\{0\}$ perpendicular to $\psi\left(C^{\Delta}(\hat{\Sigma})^{\Lambda}\right)$ with respect to the $L^{2}$-inner product. But this would contradict the density of $\psi\left(C^{\Delta}(\hat{\Sigma})^{\Lambda}\right)$. Hence $\psi\left(C^{\Delta}(\hat{\Sigma})^{\Lambda}\right)=$ $C^{\Delta}(X)^{M}$ and the theorem is proved.

Theorem 7.6 Let $X$ be a compact simply connected Riemannian manifold which is the base of a Riemannian submersion $\pi: V \rightarrow X$, where $V$ is a separable Hilbert space and let $M$ be a connected properly immersed isoparametric submanifold of $X$ with flat sections. Then $M$ defines a global foliation of $X$ and $C^{\Delta}(X)^{M}$ is isomorphic to the polynomial algebra $\mathbb{R}\left[X_{1}, \ldots, X_{k}\right]$, where $k=$ codim $M$. As free generators of the algebra $C^{\Delta}(X)^{M}$ one may take eigenfunctions of the Laplacian of $X$.

Proof: We retain the notations of the last theorem and its proof. According to Theorem 5.5, $M$ defines a global foliation of $X$. In particular we may assume that $M$ is embedded. Since $X$ is simply connected, $\hat{M}$ is connected as well and points $x, y \in \hat{\Sigma}=\hat{p}+\nu_{\hat{p}} \hat{M}$ are equivalent if and only if they lie on the same parallel manifold of $\hat{M}$ and this in turn is precisely the case if they lie on the same $W$-orbit ([T2], Theorem 9.6). Therefore $\Lambda=W, W$ is an affine Weyl group which acts cocompactly on $\hat{\Sigma}$, and $C^{\Delta}(\Sigma)^{M}=C^{\Delta}(\hat{\Sigma})^{W}$. Let $\Gamma$ be the lattice of translations of $W$ and $\bar{W}$ the isotropy group of $W$ at zero. Recall that zero is assumed to be a special point, i.e. a point through which passes a focal hyperplane from each family of parallel ones. Then $W$ is the semidirect product of $\Gamma$ with $\bar{W}$ and $C^{\Delta}(\hat{\Sigma})^{W} \otimes \mathbb{C}=\left\{\sum_{\omega \in \Gamma^{*}} a_{\omega} e^{2 \pi i \omega} \mid a_{\omega} \in \mathbb{C}, a_{\omega}=0\right.$ except for finitely many $\omega\}^{\bar{W}}$. According to [Bou1], Ch VI, $\S 3$ Théorème 1, this ring is an algebra, isomorphic to $\mathbb{C}\left[X_{1}, \ldots, X_{k}\right]$. Moreover the generators of this algebra may be chosen in a special way as follows.

Since $W$ is an affine Weyl group, there exists a root system $R$ in the dual of $\hat{\Sigma}$ such that the reflection hyperplanes are the sets $H_{\alpha, l}:=\{x \in \hat{\Sigma} \mid \alpha(x)=l\}$ where $\alpha \in R$ and $l \in \mathbb{Z}$ (Necessarily $R=\left\{ \pm 2 \alpha_{i} \mid i=1, \ldots, r\right\}$ ). Let $\Gamma$ as before denote the lattice of translations of $W=\Lambda$. Identifying translations $x \mapsto x+v$ of $\hat{\Sigma}$ with $v \in \hat{\Sigma}, \Gamma$ coincides with the lattice $Q(\check{R})=\left\{\Sigma m_{\alpha} \check{\alpha} \mid m_{\alpha} \in \mathbb{Z}, \alpha \in R\right\}$ of inverse roots (cf. [Bou1], [BD]). Hence $\Gamma^{*}$ coincides with the dual lattice $Q(\check{R})^{*}$ of $Q(\check{R})$, which is by definition the lattice of weights. By choosing a Weyl chamber one distinguishes a basis $\bar{\omega}_{1}, \ldots, \bar{\omega}_{k}$ of the lattice $\Gamma^{*}$ of weights whose elements are called the fundamental weights. There is a partial order on the set of weights. In particular $\omega^{\prime}<\omega$ iff $\omega^{\prime}$ is contained in the convex hull of $W \omega$ but not in $W \omega$ itself. Note that $W$ acts on $\Gamma$ and hence on $\Gamma^{*}$ as well. If $\omega \in \Gamma^{*}$ then the so called symmetric sum $S\left(e^{2 \pi i \omega}\right):=\sum_{\tilde{\omega} \in W \omega} e^{2 \pi i \tilde{\omega}}$ is an element of $C^{\Delta}(\hat{\Sigma})^{W} \otimes \mathbb{C}$. Now, the above quoted theorem states moreover that any elements of the form $S\left(e^{2 \pi i \bar{\omega}_{j}}\right)+\sum_{\substack{\omega \in \Gamma^{*} \\ \omega<\bar{\omega}_{j}}} a_{\omega} S\left(e^{2 \pi i \omega}\right), j=1, \ldots, k$, are a basis of the polynomial algebra 
$C^{\Delta}(\hat{\Sigma})^{W} \otimes \mathbb{C}$.

We denote the $\mathbb{C}$-linear extension of $\hat{\delta}: C^{\Delta}(\hat{\Sigma})^{W} \rightarrow C^{\Delta}(\hat{\Sigma})^{W}$ to the complexification also by $\hat{\delta}$. This leaves for all $j \in\{1, \ldots, k\}$ the finite dimensional subspaces invariant which are spanned by $S\left(e^{2 \pi i \bar{\omega}_{j}}\right)$ and the $S\left(e^{2 \pi i \omega}\right)$ with $\omega<\bar{\omega}_{j}$, as $\operatorname{supp}_{\Gamma} \hat{\delta} f$ is contained in the convex hull of $\operatorname{supp}_{\Gamma} f$ for all $f \in C^{\Delta}(\hat{\Sigma})^{W}$. Since $\hat{\delta}$ can be diagonalized on these subspaces, there exist eigenfunctions $\varphi_{j}$ of $\hat{\delta}$ of the form $\varphi_{j}=S\left(e^{2 \pi i \bar{\omega}_{j}}\right)+\sum_{\omega<\bar{\omega}_{j}} \alpha_{\omega} S\left(e^{2 \pi i \omega}\right)$ whose extensions $\tilde{\varphi}_{j}:=\psi\left(\varphi_{j}\right)$ to $X$ are thus complex valued eigenfunctions of $\Delta^{X}$. If $-\bar{\omega}_{j} \in W \bar{\omega}_{j}$, then $S\left(e^{2 \pi i \bar{\omega}_{j}}\right)$ is real valued. Eventually replacing $\varphi_{j}$ by its real part, which is of the same form $S\left(e^{2 \pi i \bar{\omega}_{j}}\right)+$ lower order terms, we may assume that $\varphi_{j}$ and hence $\tilde{\varphi}_{j}$ are real valued in this case. In general however, $-\bar{\omega}_{j}$ will not be contained in $W \bar{\omega}_{j}$. But there exists a permutation $\pi$ of $\{1, \ldots, k\}$ of order two such that $-\bar{\omega}_{j} \in W \bar{\omega}_{\pi(j)}$ for all $j=1, \ldots, k$ (cf. [Bou1],[BD]). We may arrange the fundamental weights in such a way that $\pi(i)=i$ for $i=1, \ldots, s$ and $\pi(s+i)=s+i+t$ for $i=1, \ldots, t$, where $s, t$ are nonnegative integers with $s+2 t=k$. Then we may take $\varphi_{1} \ldots, \varphi_{s+t}, \bar{\varphi}_{s+1}, \ldots, \bar{\varphi}_{s+t}$ as a basis for $C^{\Delta}(\hat{\Sigma})^{W} \otimes \mathbb{C}$ and hence also $\psi_{1}, \ldots, \psi_{k}$ where $\psi_{i}$ denotes the real part of $\varphi_{i}$ for $i=1, \ldots, s+t$ and $\psi_{s+t+i}$ denotes the imaginary part of $\varphi_{s+i}$ for $i=1, \ldots, t$. Thus $\psi_{1}, \ldots, \psi_{k}$ are real valued eigenfunctions of $\hat{\delta}$. Their extensions $\tilde{\psi}_{1}, \ldots, \tilde{\psi}_{k}$ are therefore real valued eigenfunctions of $\Delta^{X}$ and form a basis of the polynomial algebra $C^{\Delta}(X)^{M}$.

Remark: In the non-simply connected case $C^{\Delta}(X)^{M}$ is not a polynomial algebra in general. For example if $X=\mathbb{R}^{n} / \Gamma$ is a flat torus and $M$ a point, then $C^{\Delta}(X)^{M} \otimes \mathbb{C}=$ $C^{\Delta}(X) \otimes \mathbb{C}$ is the space of finite Fourier series with complex coefficients and thus isomorphic to $\mathbb{C}\left[X_{1}, \ldots, X_{n}, X_{1}^{-1}, \ldots, X_{n}^{-1}\right]$.

Corollary 7.7 Let $X$ and $M$ be as in the last theorem. Then there exists a mapping $\psi: X \rightarrow \mathbb{R}^{k}$ whose components $\psi_{i}: X \rightarrow \mathbb{R}$ are eigenfunctions of the Laplacian of $X$ and whose level sets are the leaves of the isoparametric foliation determined by $M$. Moreover $\psi$ is a submersion when restricted to the set of regular leaves, i.e. to those of maximal dimension.

Proof: We let $\psi:=\left(\psi_{1}, \ldots, \psi_{k}\right)$ with $\psi_{i}$ as in the proof of the theorem. Then the $\psi_{i}$ are eigenfunctions of $\Delta^{X}$ and are constant on the parallel manifolds $M_{\xi}$ of $M$. Since $C^{\infty}(\hat{\Sigma})^{W}$ separates the $W$-orbits, $C^{\infty}(X)^{M}$ separates the parallel manifolds of $M$. That is, for any $M_{\xi_{1}} \neq M_{\xi_{2}}$ there exists an $f \in C^{\infty}(X)^{M}$ with $f\left(M_{\xi_{1}}\right) \neq f\left(M_{\xi_{2}}\right)$. Due to the density of $C^{\Delta}(X)^{M}$ in $C^{\infty}(X)^{M}$ with respect to the supremum norm we may choose such an $f$ from $C^{\Delta}(X)^{M}$. As $C^{\Delta}(X)^{M}$ is generated by the $\psi_{i}$ as an algebra, we find even an index $i$ with $\psi_{i}\left(M_{\xi_{1}}\right) \neq \psi_{i}\left(M_{\xi_{2}}\right)$. Hence $\psi\left(M_{\xi_{1}}\right) \neq \psi\left(M_{\xi_{2}}\right)$ and the level sets of $\psi$ are the parallel manifolds of $M$.

If $x \in \hat{\Sigma}_{r e g}$ and $v \in T_{x} \hat{\Sigma}, v \neq 0$, then there exists an $f \in C^{\infty}(\hat{\Sigma})^{W}$ with $<\operatorname{gradf}_{x}, v>\neq 0$. In fact, one can extend any $C^{\infty}$-function with support in a suffi- 
ciently small neighborhood of $x$ to a $W$-invariant $C^{\infty}$-function. We may approximate $f$ and its first derivatives uniformly by functions from $C^{\Delta}(\hat{\Sigma})^{W}$. Thus there exists even an $f \in C^{\Delta}(\Sigma)^{W}$ with $\left\langle\operatorname{gradf}_{x}, v>\neq 0\right.$. The extension $\tilde{f}$ of $f$ to $X$ lies in $C^{\Delta}(X)^{M}$ and satisfies $\tilde{f} \circ \pi_{\left.\right|_{\hat{\Sigma}}}=f$ and hence $<\operatorname{grad} \tilde{f}_{\pi(x)}, \pi_{*} v>=<\operatorname{gradf} f_{x}, v>\neq 0$. As the $\psi_{i}$ generate $C^{\Delta}(X)^{M}$ as an algebra there exists therefore an index $i$ with $<\left(\operatorname{grad} \psi_{i}\right)_{\pi(x)}, \pi_{*} v>\neq 0$ which implies the surjectivity of $\psi_{*}$ at $\pi(x)$.

In the example $X=G$, a compact Lie group with biinvariant metric, and $M$ a conjugacy class of a regular element the theorems and the corollary are of course well known and are weak versions of fundamental results in the theory of representations of compact Lie groups. In fact, the action of $G$ on itself by conjugation is hyperpolar with the maximal tori as flat sections. If $T$ is a maximal torus, then $C^{\infty}(G)^{M}=$ $C^{\infty}(G)^{G}$ is the set of smooth class functions on $G$ and $C^{\infty}(T)^{M}=C^{\infty}(T)^{W_{G}}$, where $W_{G}$ denotes the Weyl group of $G$. Moreover, $C^{\Delta}(G)$ is spanned by the coefficients of representations of $G$ (Peter-Weyl) and $C^{\Delta}(G)^{G} \otimes \mathbb{C}$ is the complex vector space spanned by the characters of representations of $G$. Thus $C^{\Delta}(G)^{G} \otimes \mathbb{C}$ is isomorphic to $R(G) \otimes \mathbb{C}$, where $R(G)$ denotes the representation ring of $G$. Hence Theorem 7.1 shows in this case that restriction from $G$ to $T$ defines an isomorphism $R(G) \otimes \mathbb{C} \rightarrow(R(T) \otimes \mathbb{C})^{W_{G}}$. Moreover, if $G$ is simply connected, Theorem 7.6 yields that $R(G) \otimes \mathbb{C}$ is a polynomial algebra in $k=$ rank $G$ free generators. From representation theory one actually knows $R(G) \cong \mathbb{Z}\left[\chi_{\varrho_{1}}, \ldots, \chi_{\varrho_{k}}\right]$, where $\varrho_{j}, j=1, \ldots, k$, is the representation with highest weight $\bar{\omega}_{j}$, and that $\chi_{\varrho_{j}} \circ \pi_{\left.\right|_{\hat{\Sigma}}}=S\left(e^{2 \pi i \bar{\omega}_{j}}\right)+$ lower order terms.

The discussion of the last example shows that isoparametric submanifolds are intimately related to the representation theory of compact Lie groups. This becomes even more evident by Corollary 7.7. In fact, if $M$ is a compact isoparametric submanifold with flat sections of a compact simply connected normal homogeneous space $G / H$, then $M$ is the level set of a function $\psi=\left(\psi_{1}, \ldots, \psi_{k}\right)$ whose components $\psi_{i}$ are eigenfunctions of the Laplacian. The functions $\tilde{\psi}_{i}:=\psi_{i} \circ \pi_{G}$, where $\pi_{G}: G \rightarrow G / H$ is the projection, are then eigenfunctions of the Laplacian of $G$ and hence coefficients of representations of $G$. 


\section{Appendix A}

\section{A remark on polar actions}

In this appendix we prove a result which has been conjectured by Palais and Terng in [PT1], Remark 3.3 and [PT2], 5.6.8.

Theorem A Let $X$ be a complete Riemannian manifold and $G$ a Lie group which acts properly by isometries on $X$. If the distribution of normal spaces to the regular orbits is integrable then there exists a complete totally geodesic immersed section for the action of $G$ which moreover meets all orbits and always perpendicularly.

We will recall some basic facts about existence and extendibility of totally geodesic submanifolds first and begin with the following result of Cartan.

Theorem (E. Cartan, see [Her]) Let $M$ be a Riemannian manifold, $p \in M$ and $W$ a linear subspace of $T_{p} M$. Then there exists a totally geodesic submanifold $N$ of $M$ with $p \in N$ and $T_{p} N=W$ if and only if there exists some $\epsilon \in \mathbb{R}_{+}$such that for every geodesic $\gamma$ in $M$ with $\gamma(0)=p$ and $\gamma^{\prime}(0) \in W \cap U_{\epsilon}(0)$ the Riemannian curvature tensor of $M$ at $\gamma(1)$ preserves the parallel translate of $W$ along $\gamma$ from $p$ to $\gamma(1)$.

Let $M$ be a fixed Riemannian manifold. We recall that two isometric immersions $f_{i}: N_{i} \rightarrow M, i=1,2$ are said to be equivalent, if there exists a global isometry $g: N_{1} \rightarrow N_{2}$ such that $f_{1}=f_{2} \circ g$. If $f: N^{k} \rightarrow M$ is an isometric totally geodesic immersion, then it induces a (differentiable) map $\tilde{f}: N \rightarrow G_{k}(M)$, where $G_{k}(M)$ is the Grassmannian of $k$-planes of $T M$. Namely, $\tilde{f}(x)=\left(f(x), f_{*}\left(T_{x} N\right)\right)$. We say that the isometric totally geodesic immersion $(N, f)$ is compatible, if $N$ is connected and $\tilde{f}$ is injective. Any isometric totally geodesic immersion from a connected Riemannian manifold $N$ can be factorized through a compatible one. In fact, this can be done by identifying points in $N$ with the same image in the Grassmannian. In this way the quotient space is a differentiable manifold which admits a unique Riemannian structure such that the projection from $N$ is a local isometry. This is due to the fact that locally a totally geodesic submanifold is completely determined by its tangent space at a point. By the same reason one can show the following: a compatible isometric totally geodesic immersion is completely determined, up to equivalence, by its image into the Grassmannian.

Let $\mathfrak{T}$ denote the collection of all (equivalence classes of) compatible isometric totally geodesic immersion into $M$. T has a natural partial order $\preceq$. Namely, $\left(N_{1}, f_{1}\right) \preceq$ $\left(N_{2}, f_{2}\right)$, if there is a 1-1 local isometry $i: N_{1} \rightarrow N_{2}$ such that $f_{1}=f_{2} \circ i$. In this case we say that $\left(N_{2}, f_{2}\right)$ extends $\left(N_{1}, f_{1}\right)$. It is standard to show that $\left(N_{1}, f_{1}\right) \preceq\left(N_{2}, f_{2}\right)$, if and only if $\tilde{f}_{1}\left(N_{1}\right) \subset \tilde{f}_{2}\left(N_{2}\right)$. Using Zorn's Lemma, there exists for each $(N, f) \in \mathfrak{T}$, a maximal $(\bar{N}, \bar{f}) \in \mathfrak{T}$ extending $(N, f)$. 
Lemma 1 Let $\left(N_{1}, f_{1}\right),\left(N_{2}, f_{2}\right) \in \mathfrak{T}$ and assume that $\tilde{f}_{1}\left(N_{1}\right) \cap \tilde{f}_{2}\left(N_{2}\right) \neq \emptyset$. Then there exists $(N, f) \in \mathfrak{T}$ which extends both immersions.

Proof: Define $N$ to be the disjoint union of $N_{1}$ and $N_{2}$ with the following relation: $x_{1} \sim x_{2}$ iff $\tilde{f}_{1}\left(x_{1}\right)=\tilde{f}_{2}\left(x_{2}\right)$, for $x_{1} \in N_{1}$ and $x_{2} \in N_{2}$. Observe that $N$ is connected. Let $f: N \rightarrow M$ be defined by $f\left(\left[x_{i}\right]\right)=f_{i}\left(x_{i}\right), x_{i} \in N_{i}, i=1,2$. The proof follows now in a standard way.

Lemma 2 Let $\left(N_{1}, f_{1}\right),\left(N_{2}, f_{2}\right) \in \mathfrak{T}$ with $\left(N_{1}, f_{1}\right)$ maximal and assume that there exist sequences $\left\{x_{n}\right\}$ in $N_{1}$ and $\left\{y_{n}\right\}$ in $N_{2}$ such that :

(i) $\tilde{f}_{1}\left(x_{n}\right)=\tilde{f}_{2}\left(y_{n}\right)$.

(ii) $\left\{y_{n}\right\}$ converges to some $y \in N_{2}$.

Then also $x_{n}$ converges.

Proof: Immediate from Lemma 1.

Lemma 3 Under the assumptions of the above theorem, let $p \in X$ and let $H$ be the connected component of the isotropy subgroup of $G$ at $p$. Then the isotropy representation of $H$ on the normal space $\nu_{p}(G . p)$ (i.e. the so called slice representation) is polar. Moreover, if $\Sigma$ is a (linear) section for this representation then $\exp _{p}\left(\Sigma \cap B_{\varepsilon}(0)\right)$ is locally a (totally geodesic) section for the action of $G$, for $\varepsilon$ small.

Proof: First observe that a totally geodesic connected submanifold $\tilde{\Sigma}$ of $X$ meets orbits orthogonally if and only if, for some $q \in \tilde{\Sigma}$ and any $Z \in \operatorname{Lie}(G)$ (identified with a Killing field on $X$ ), the following two conditions hold:

(i) $Z . q \in\left(T_{q} \tilde{\Sigma}\right)^{\perp}$.

(ii) $(\nabla Z)_{q}\left(T_{q} \tilde{\Sigma}\right) \subset\left(T_{q} \tilde{\Sigma}\right)^{\perp}$.

In fact, if $\gamma(t)$ is a geodesic in $\tilde{\Sigma}$ starting at $q$ then the Jacobi field $J(t)=Z \cdot \gamma(t)$ has initial conditions $J(0)=Z . q, J^{\prime}(0)=\nabla_{\gamma^{\prime}(0)} Z$ which are both in $\left(T_{q} \tilde{\Sigma}\right)^{\perp}$.

Choose a sequence $\left\{p_{n}\right\}$, in the regular points of $M$ for the action of $G$, such that $p_{n} \rightarrow p$ and let $\tilde{\Sigma}_{n}$ be local sections for the action of $G$ with $p_{n} \in \tilde{\Sigma}$. By choosing, eventually, a subsequence we may assume that $T_{p_{n}} \tilde{\Sigma}=\nu_{p_{n}}$ converges to some subspace $W$ of $T_{p} X$, which must be orthogonal to $T_{p}(G . p)$. By continuity we obtain, in particular, that $(\nabla Z)_{q}(W) \subset(W)^{\perp}$ for all $Z \in \operatorname{Lie}(H)$. Since $\left.\frac{d}{d t}\right|_{0}(\operatorname{expt} Z)_{* p}=(\nabla Z)_{p}$, we have that the Lie algebra $\mathfrak{h}$ of the image of the isotropy representation of $H$ at $p$ coincides with $\left\{(\nabla Z)_{p}: Z \in \operatorname{Lie}(H)\right\}$. So, $\mathfrak{h}(W) \subset W^{\perp}$, which implies the polarity of the slice representation (since the codimension of the action of $G$ is the same as the codimension of the slice representation). Let now $\Sigma \subset T_{p} X$ be any section of the isotropy action. We want to show that the image by $e x p_{p}$, of some open neighborhood of $0 \in \Sigma$, is a totally geodesic submanifold of $X$. We will need the following auxiliary result which is well known and standard to prove. 
Sublemma There exists $\varepsilon>0$ such that the codimension of G.exp $(v)$ in $X$ is equal to the codimension of H.v in $\nu_{p}(G . P)$, for all $v \in \Sigma$ with $\|v\|<\varepsilon$. In particular, if $v$ is a principal vector for the slice representation with $\|v\|<\epsilon$, then the geodesic $\gamma_{v}(t)$, $0 \neq t \in[-1,1]$, consists of principal points for the action of $G$ on $X$.

Proof of Lemma 3 (continued): Let $\varepsilon>0$ be given by the above sublemma which we may assume smaller than the injectivity radius at $p$. Let $v \in \Sigma$ be a principal vector for the isotropy representation with $\|v\|<\varepsilon$ and consider the geodesic $\gamma_{v}$. Observe that $\gamma^{\prime}(t) \in \nu_{\gamma(t)}$ for all $t \in(0,1]$. In fact, if $Z \in \operatorname{Lie}(G)$ then $\left\langle Z \cdot \gamma_{v}(0), \gamma_{v}^{\prime}(0)\right\rangle=0$ and $\left.\frac{d}{d t}\right|_{0}\left\langle Z \cdot \gamma_{v}(t), \gamma_{v}^{\prime}(t)\right\rangle=\left\langle\nabla_{\gamma_{v}^{\prime}(0)} Z, \gamma_{v}^{\prime}(0)\right\rangle=0$, by the Killing equation.

We have that $\nu^{t}:=\nu_{\gamma_{v}(t)}, 0<t \leq 1$, is parallel along $\gamma_{v}(t)$, since $\nu$ is a totally geodesic distribution. It is standard to show that the distance $d\left(\nu^{t}, T_{\gamma_{v}(t)}\left(\exp _{p}(\Sigma \cap\right.\right.$ $\left.\left.B_{\varepsilon}(0)\right)\right)$ ) tends to zero, if $t \rightarrow 0$ (regarding, for instance, both subspaces as elements of $\Lambda^{k}\left(T_{\gamma_{v}(t)} X\right)$, where $\left.k=\operatorname{dim}(\nu)\right)$. Roughly speaking $T_{\gamma_{v}(t)}\left(\exp _{p}\left(\Sigma \cap B_{\varepsilon}(0)\right)\right)$ approximates the normal space $\nu^{t}$ of the orbit $G \cdot \gamma_{v}(t)$, for $t$ small, $t \neq 0$. So, if we set $\nu^{0}=\Sigma$, then $\nu^{t}, t \in[0,1]$, is a parallel distribution along $\gamma_{v}$. Since $\nu$ is totally geodesic, we have in addition that $\nu^{t}$ is invariant under the curvature tensor $R$ of $X$, for $t \neq 0$ and by continuity for all $t \in[0,1]$. Since principal vectors are dense in $\Sigma$ we obtain, by a continuity argument, that for any $v \in \Sigma$ with $\|v\|<\varepsilon$ the parallel transport of $\Sigma$ along the geodesic $\gamma_{v \mid[0,1]}$ is invariant under the curvature tensor $R$. Then $\exp _{p}\left(\Sigma \cap B_{\varepsilon}(0)\right)$ is a totally geodesic submanifold of $X$ due to the Theorem of Cartan. Observe that the parallel transport of $\Sigma$ along the geodesic $\gamma_{v \mid[0,1]}, v \in \Sigma$ principal, coincides with $T_{\gamma_{v}(1)}\left(\exp _{p}\left(\Sigma \cap B_{\varepsilon}(0)\right)\right)$. By construction, this parallel transport must also coincide with $\nu_{\gamma_{v}(1)}$. So, $\exp _{p}\left(\Sigma \cap B_{\varepsilon}(0)\right)$ is a local section.

Proof of Theorem A: Let $f: N \rightarrow X$ be a maximal isometric totally geodesic immersion which extends some local section. Observe that $(N, f)$ meets orbits perpendicularly, since it does so in an open non empty subset (using the fact that Killing fields are Jacobi along geodesics). If $(N, f)$ is not complete there exists a geodesic $\gamma:[0,1) \rightarrow N$ and a sequence $\left\{t_{n}\right\}$ in $[0,1)$ which tends to 1 and such that $\left\{\gamma\left(t_{n}\right)\right\}$ is not convergent in $N$. We have that $f_{*}\left(T_{\gamma\left(t_{n}\right)} N\right)$ converges to $W$, the parallel transport along $\beta$ of $f_{*}\left(T_{\gamma(0)} N\right)$, where $\beta:[0,1] \rightarrow X$ is the geodesic extending $f \circ \gamma$. By Lemma 3 and its proof we have that $W$ is a section of the isotropy representation at $\beta(1)$. Moreover, $\exp _{\beta(1)}\left(W \cap B_{\varepsilon}(0)\right)$ is a totally geodesic submanifold of $X$, if $\varepsilon$ is small. If $n$ is large, one has that $\left(\exp _{\beta(1)}\right)_{* v_{n}}\left(T_{v_{n}} W\right)=f_{* \gamma\left(t_{n}\right)}\left(T_{\gamma\left(t_{n}\right)} N\right)$, where $v_{n}=\left(1-t_{n}\right)\left(-\beta^{\prime}(1)\right)$ (since both subspaces are parallel along $\beta$ ). Then one has, for the induced maps on the Grassmannian, that $\tilde{f}\left(\gamma\left(t_{n}\right)\right)=e \tilde{x} p_{\beta(1)}\left(v_{n}\right)$. Since $v_{n}$ converges to 0 and $\gamma\left(t_{n}\right)$ does not converge, we conclude, by Lemma 2 , that $(N, f)$ is not maximal. A contradiction. Thus $N$ is complete. Observe that $f(N)$ meets every orbit, as $G$-orbits are closed (the action is proper) and so, the exponential of the normal space to any orbit meets all other orbits. 
Remark: After finishing this paper we learned that K. Grove and W. Ziller gave an independent proof of Theorem A. This will appear in [GZ]. Moreover G. Thorbergsson draw our attention to a paper by $\mathrm{H}$. Boualem [Bo] which treats the more general case of singular riemannian foliations and contains Theorem A as a special case. But we hope that our shorter proof in the particular situation will still be of some interest.

\section{Appendix B}

\section{Isoparametric submanifolds in Hilbert spaces are embedded and have globally flat normal bundle}

Terng has shown that isoparametric submanifolds of euclidean space have globally flat normal bundle ([T1], Proposition 3.6). The aim of this appendix is to generalize this result to infinite dimensions by a slight modification of her arguments. Moreover we fill a gap in the proof of Corollary 7.2 of [T2] which says that isoparametric submanifolds of Hilbert spaces are embedded. Both problems are related, and will be resolved simultanously. Thus we are going to prove:

Theorem B Let $V$ be a separable Hilbert space and $f: M \rightarrow V$ a proper Fredholm immersion of finite codimension which satisfies

(i) $\nu M$ is flat, and

(ii) for any parallel normal vector field $\xi(t)$ along any differentiable curve $c:[0,1] \rightarrow M$, the shape operators $A_{\xi(0)}$ and $A_{\xi(1)}$ are orthogonally equivalent.

If $M$ is connected, then $f(M)$ is an embedded submanifold of $V$ with globally flat normal bundle. In particular $M$ (more precisely $f$ ) itself has globally flat normal bundle and $M$ is isoparametric according to the definition of Terng [T2].

Remark: One has to be a little bit careful with the notion of globally flat normal bundle of an immersion $f: M \rightarrow X$. Even if $f(M)$ is embedded this depends strongly on $f$. For example, if $\nu M$ has finite holonomy (as in our case, see the first lines of the proof of Theorem B) one can replace $M$ by a finite cover such that the new immersion has globally flat normal bundle. This shows in particular that the assumption (i) above may be replaced by global flatness of $\nu M$.

We are going to deduce Theorem B from the following result of Terng which seems to us the correct statement of what she proved in [T2] about the embeddedness of isoparametric submanifolds. Namely, to show that an immersed isoparametric submanifold $f: M \rightarrow V$ of a Hilbert space is embedded, Terng proves that each critical point of the euclidean distance function (to a non-focal point) has a Bott-Samelson cycle and that therefore $f$ ist taut (Theorem 7.1 of [T2]) and hence an embedding (Corollary 
5.9 of [T2]). However, the construction of the Bott-Samelson cycles only works if $f$ is injective on all curvature spheres (cf. the proof of Lemma 8.3.2 in [PT2]). While the injectivity is clear for curvature spheres of dimension at least 2 (as they are simply connected and the restriction of $f$ is a local isometry onto a round sphere), it is in general not true for 1-dimensional curvature circles.

Theorem (Terng [T2]) Let $V$ be a separable Hilbert space and $f: M \rightarrow V$ a proper Fredholm immersion of finite codimension which is isoparametric in the sense of Terng, i.e. satisfies

(i) $\nu M$ is globally flat, and

(ii) for any parallel normal vector field $\xi$ on $M$, the shape operators $A_{\xi(p)}$ and $A_{\xi(q)}$ are orthogonally equivalent for all $p, q \in M$.

If $M$ is connected and $f$ is injective on all curvature circles, then $f$ is an embedding.

\section{Remarks:}

(i) We are grateful to G. Thorbergsson who pointed out to us another proof of this theorem using only the mountain pass lemma, ([Th]).

(ii) If all multiplicities of $M$ (i.e. the dimensions of the curvature spheres) are at least 2, then Theorem B is a direct consequence of Terng's result and the remark following Theorem B.

(iii) Under the assumptions of Terng's theorem the parallel immersions $f_{\xi}: M \rightarrow$ $V, p \mapsto f(p)+\xi(p)$, are also embeddings for all non-focal $\xi \in \nu(M)$. In fact, this new immersion has also globally flat normal bundle and is injective on all curvature spheres as well (since along a curvature sphere $\xi(p)=\lambda(f(p)-m)+\xi_{0}$ for some $\lambda \in \mathbb{R}$, and $m, \xi_{0} \in V$ and hence $\left.f_{\xi}(p)=(1+\lambda) f(p)-\lambda m+\xi_{0}\right)$.

Proof of Theorem B: We may assume that $M$ is full and that therefore $\nu_{x} M$ is spanned for any $x \in M$ by the curvature normals $n_{i}(x), i \in I$. These are a well defined set of normal vectors at each point, invariant under parallel translation, but not necessarily of globally defined vector fields. Let $x_{0} \in M$ and $S:=\left\{n_{i}\left(x_{0}\right) /\left\|n_{i}\left(x_{0}\right)\right\| \geq\right.$ $r$ \} for some $r>0$. Since $M$ is full and of finite codimension we may assume that $r$ is big enough, so that $S$ spans $\nu_{x_{0}}(M) . S$ is a finite set, because the shape operators are compact. Thus the holonomy group of $\nu M$ at $x_{0}$, which preserves $S$, is finite. Moreover it is trivial if the curvature normals have pairwise different length.

To get theorem B from Terng's result (by going to a finite cover of $M$ we easily could assume that $\nu M$ is globally flat) we want to factorize $f$ over an appropriate quotient but run then into the difficulty that the normal bundle of the quotient might not be globally flat any more. We therefore assume first that the curvature normals $n_{i}$ have different lengths for all $i \in I$. Hence $\nu(M)$ is globally flat and the $n_{i}$ are globally defined. We fix $i \in I$ and let $S_{i}(q)$ denote the curvature sphere through $q \in M$, which is by definition the integral manifold of the curvature distribution $E_{i}$. Then $f\left(S_{i}(q)\right)$ is

a round sphere of radius $\frac{1}{\left\|n_{i}\right\|}$ for all $q \in M$. We assume $\operatorname{dim} S_{i}(q)=1$ in addition and 
define an equivalence relation $\sim$ on $M$ by $x \sim y$ iff $f(x)=f(y)$ and $y \in S_{i}(x)$. Let $k(x)$ denote the number of points equivalent to $x$. Thus the closed geodesic $S_{i}(x)$ covers the circle $f\left(S_{i}(x)\right) \quad k(x)$-times under $f$. We fix $x \in M$ and orient $S_{i}(x)$ by choosing a tangent vector field $Z$ of constant length $2 \pi /\left\|n_{i}\right\|$. This vector field can be extended to a vector field $\tilde{Z}$ on a neighborhood $U$ of $S_{i}(x)$ consisting of integral manifolds of $E_{i}$ with $\left\|\tilde{Z}_{i}\right\| \equiv 2 \pi /\left\|n_{i}\right\|$ and $\tilde{Z}(y) \in E_{i}(y)$ for all $y \in U$. Let $\varphi^{t}: U \rightarrow U, t \in \mathbb{R}$, be the associated flow and $\varphi:=\varphi^{1}$. Then $f\left(\varphi^{t}(y)\right)$ covers the circle $f\left(S_{i}(y)\right)$ just once if $t$ runs from 0 to 1. In particular $f(\varphi(y))=f(y)$ for all $y \in U$ and hence $f\left(\varphi^{k(x)} y\right)=f(y)$. Since $\varphi^{k(x)}(x)=x$ and $f$ is locally injective there exists a neighborhood $U^{\prime}$ of $x$ with $\varphi^{k(x)}(y)=y$ and hence with $k(y) \leq k(x)$ for all $y \in U^{\prime}$. By shrinking $U^{\prime}$ further we then find also a neighborhood $U^{\prime \prime}$ with $k(y)=k(x)$ for all $y \in U^{\prime \prime}$, as otherwise there would exist a sequence $y_{n}$ converging to $x$ with $k\left(y_{n}\right)=l<k(x)$ implying $f\left(\varphi^{l} x\right)=f(x)$ which is a contradiction. Thus $k(x)$ is locally constant and hence constant on $M$ by connectedness. This implies that $M / \sim$ is locally given as the quotient of $M$ by the free action of the group $\left\{\varphi^{l} \mid l=1, \ldots, k\right\}$ of diffeomorphism and is hence in a natural way a manifold such that $M \rightarrow M / \sim$ becomes a $k$-fold covering (cf. [Bou2], ch. 5.9.5). Furthermore $f$ factorizes through $M / \sim$. Since the fibres of $f$ are finite, there are only finitely many indices $i \in I$, such that $S_{i}$ is a curvature circle of $M$ with more than one equivalent point. Thus repeating the above process finitely many times one gets a quotient manifold $\bar{M}$ and an induced isoparametric immersion $\bar{f}: \bar{M} \rightarrow V$ whose normal bundle is still globally flat (as the curvature normals have different lengths) and which is injective on all curvature circles. Thus $\bar{f}$ is an embedding by Terng's theorem and $f(M)=\bar{f}(\bar{M})$ is an embedded submanifold with globally flat normal bundle.

In the general situation (where the $\left\|n_{i}\right\|$ are not necessarily pairwise different) we choose $q \in M$ and $\xi \in \nu_{q} M$ such that $\left\langle\xi, n_{i}(q)>\neq 1\right.$ and

$$
\left\|\frac{n_{i}(q)}{1-<\xi, n_{i}(q)>}\right\| \neq\left\|\frac{n_{j}(q)}{1-<\xi, n_{j}(q)>}\right\|
$$

for all $i, j \in I$ and $j \neq i$. This can be done since $I$ is countable. Let $M_{\xi}:=\{(p, \tilde{\xi}) \in$ $M \times V \mid \tilde{\xi} \in \nu_{p} M$ is obtained by parallel translating $\xi$ in $\nu M$ along any curve in $M$ connecting $q$ with $p\}$ and $f_{\xi}: M_{\xi} \rightarrow V$ the mapping defined by $f_{\xi}(p, \tilde{\xi})=f(p)+\tilde{\xi}$. Then $f_{\xi}$ is a proper Fredholm immersion of finite codimension which satisfies (i) and (ii) of the theorem. Moreover the curvature normals of $M_{\xi}$ at $(q, \xi)$ are exactly

$$
\frac{n_{i}(q)}{1-<\xi, n_{i}(q)>}, i \in I,
$$

and therefore have different lengths. Thus $f_{\xi}\left(M_{\xi}\right)$ is an embedded submanifold of $V$ with globally flat normal bundle by the first step. The vector $-\xi \in \nu_{(q, \xi)} M_{\xi}$ defines a global parallel normal field along $M_{\xi}$ and hence also along $f_{\xi}\left(M_{\xi}\right)$. The corresponding 
parallel manifold is $f(M)$. By the remark (iii) following Terng's theorem, $f(M)$ is therefore an embedded submanifold with globally flat normal bundle.

\section{References}

[BC] Bishop, R.L. and Crittenden, R.J., Geometry of manifolds, Academic Press, New York, 1964

[BD] Bröcker, Th. and tom Dieck, T., Representations of compact Lie groups, Springer 1995

[Bo] Boualem, H., Feuilletages riemanniennes singuliers transversalement integrables, Compositio Math. 95, 101 - 125

[Bou1] Bourbaki, N., Groupes et algèbres de Lie, Chapitre 4,5 et 6, Hermann, 1968

[Bou2] Bourbaki, N., Variétés différentielles et analytiques, Hermann 1967

[Ca] Cartan, E., Familles de surfaces isoparamétriques dans les espaces à courbure constante, Annali di Mat. 17 (1938), 177 - 191

[CW] Carter, S. and West, A., Isoparametric systems and transnormality, Proc. London Math. Soc., 51 (1985), 520 - 542

[DS] Dunford, N. and Schwarz, J.T., Linear Operators, Part II, Interscience Publishers Inc. New York, 1958

[GW1] Gromoll, D. and Walschap, G., Metric fibrations in euclidean space, Asian J. Math. 1 (1997), 716 - 728

[GW2] Gromoll, D. and Walschap, G., The metric fibrations in euclidean space, preprint [GZ] Grove, K. and Ziller, W., Characterizations of Polar actions, in preparation

[Ha] Harle, C.E., Isoparametric families of submanifolds, Bol. Soc. Bras. Math. 13 (1982), $35-48$

[Hel1] Helgason, S., Invariants and fundamental functions, Acta Math. 109 (1963), 241 $-258$

[Hel2] Helgason, S., Groups and Geometric Analysis, Academic Press, 1984

[Her] Hermann, R., Existence in the large of totally geodesic submanifolds of Riemannian spaces, Bull. Amer.Math. Soc. 66 (1960), 59-61

[HPTT] Heintze, E., Palais, R.S., Terng, C.L. and Thorbergsson, G., Hyperpolar Actions on Symmetric Spaces, Geometry, Topology \& Physics for Raoul Bott, ed. by S.T. Yau, (1995), 214 - 245

[K] Kato, T., Perturbation theory for linear operators, Springer 1966

[Ko] Kollross, A., A classification of hyperpolar and cohomogeneity one actions, PhD thesis, Augsburg 1998

[KT] King, C. and Terng, C.L., Minimal submanifolds in path space, Global Analysis in Modern Mathematics, ed. by K. Uhlenbeck, Publisch or Perish, 1993, 253 - 282 [M] Milnor, J., Morse Theory, Princeton University Press, Princeton, 1963 
[O'N1] O'Neill, B., The fundamental equations of submersions, Michigan Math. J. 13 (1966), $459-469$

[O'N2] O'Neill, B., Submersions and geodesics, Duke Math. J. 34 (1967), 363 - 373

[PT1] Palais, R.S. and Terng, C.L. A general theory of canonical forms, Trans. Amer. Math. Soc. 300 (1987), 771 - 789

[PT2] Palais, R. and Terng, C.-L., Critical Point Theory and Submanifold geometry, Lect. Notes in Math. 1353, Berlin Heidelberg New York: Springer 1988

[PTh] Podestà, F. and Thorbergsson, G., Polar actions on rank one symmetric spaces, preprint

[T1] Terng, C.L., Isoparametric Submanifolds and their Coxeter Groups, J. Differential Geometry 21 (1985), 79 - 107

[T2] Terng, C.L., Proper Fredholm Submanifolds of Hilbert Space, J. Differential Geometry 29 (1989), 9 - 47

[T3] Terng, C.L., Polar actions on Hilbert space, J. Geom. Anal. 5 (1995), 129 - 150

[Th] Thorbergsson, G., private communication, May 1999

[TT] Terng, C.L. and Thorbergsson, G., Submanifold geometry in symmetric spaces, J. Differential Geometry 42 (1995), 665 - 718

[W] Wang, Q., Isoparametric hypersurfaces in complex projective spaces, 1980 Beijing Symposium on Diff. Geom. and Diff. Eq., Vol. 3, 1509 - 1524, Science Press, Beijing 1982

Ernst Heintze

Institut für Mathematik

Universität Augsburg

Universitätsstrasse 14

D - 86159 Augsburg, Germany

heintze@math.uni-augsburg.de
Xiaobo Liu

Department of Mathematics University of Notre Dame

Notre Dame, IN 46556-0398

USA

xliu3@hilbert.helios.nd.edu

\author{
Carlos Olmos \\ Facultad de Matemática, Astronomía y Física \\ Universidad Nacional Córdoba \\ Medina Allende y Haya de la Torre \\ Ciudad universitaria \\ 5000. Córdoba, Argentina \\ olmos@mate.uncor.edu
}

